\title{
Connections and Effective $S$-Matrix in Triangle Representation for Quantum Scattering
}

\author{
YU. A. KUPERIN* \\ Istituto Nazionale di Fisica Nucleare, Sezione Sanita, \\ Viale Regina Elena 299, 1-00161, Rome, Italy
}

AND

\author{
P. B. Kurasov, Yu. B. Melnikov, and S. P. Merkuriev \\ Institute for Physics, Leningrad University, \\ Leningrad 198904, USSR
}

Received September 15, 1989; revised May 4, 1989

\begin{abstract}
For quantum few-body systems, a method of spectral representation generated by specially extracted sub-Hamiltonians is developed. In this approach, the connections between the geometry of a base manifold, the spectral structure of sub-Hamiltonians, and the geometric characteristics of induced Hilbert bundles are investigated. The appropriate relations between connections on these Hilbert bundles and global invariants of $S$-matrix are obtained. Some applications of the proposed approach to exactly solvable two- and three-body scattering problems are considered. 1991 Academic Press, Inc.
\end{abstract}

\section{INTRODUCTION}

The increasing interest in the geometrical aspects of the quantum scattering theory can be explained by several reasons of both theoretical and computational nature. The first of them is mainly connected with the fact [1-3] that spectral representation of a specially extracted sub-Hamiltonian in the $N$-body quantummechanical problem and further averaging over the eigenfunctions of this subHamiltonian generate $(N-1)$-body dynamical equations. These equations describe the common dynamics of quasi-particles and some classical gauge fields, generated by the procedure of triangle (adiabatic) expansions [1-12].

On the other hand, in this approach the sub-Hamiltonian $\mathscr{L}(x)$ of the system depends on the parameter $x$, belonging to some manifold $\mathscr{M}$. The triple connections between the geometry of the manifold $\mathscr{M}$, the spectral structure of the operator $\mathscr{L}(x)$ and the geometric characteristics of the induced Hilbert bundles is an impor-

* On leave of absence from Department of Mathematical and Computational Physics, Leningrad University, USSR. 
tant subject of investigation. However, the wave functions of the operator $\mathscr{L}(x)$ continuous spectrum and, consequently, the corresponding $S$-matrices practically have not been under discussion in similar approach (except papers $[1,11,12]$ ). At the same time, there is no doubt that from the point of view of the scattering theory that the investigation of triple connections mentioned above is a most interesting problem. The special interest is caused by the systems where parameter $x \in \mathscr{M}$ is directly connected with the dynamics of the system itself. It brings the dynamical meaning to the geometry associated with the triangle expansion for such systems. The three-body system is a good example of an interesting quantum-mechanical system obeying the conditions mentioned above.

Finally, the computational reason is the following. The procedure of triangle expansion mentioned above leads to the effective scattering theory in the space of a lower dimension. The investigation of this theory is an easier problem than the analysis of the original many-body (and, consequently, many-dimensional) problem. In this sense the triangle expansion technique can be treated as a variant of dimensional reduction [11-14].

One should also notice that the decomposition of the original problem into an eigenvalue problem for the sub-Hamiltonian and effective scattering problem appearing after dimensional reduction allow us to reconstruct the interaction from the scattering data, step by step. This procedure turns the solution of the inverse problem into a considerably easier task. Thus the main problem in such an approach is to obtain the links between induced gauge fields and global invariants of the $S$-matrix.

In this paper we shall discuss the questions mentioned above in the following order. In Section 2 the general scheme of the method is discussed. We formulate the most general conditions for the system available for the analysis by the triangle expansion method in Subsection 2.1. Subsection 2.2 contains the geometrical interpretation of the method; connections on the corresponding Hilbert bundles are studied there. In Subsection 2.3 we interpret the connection operator as a gauge field, induced by the dimensional reduction procedure.

In Section 3 we consider the three-body system in $\mathbb{R}^{3}$. The effective problem for coefficients of the triangle expansions of the wave functions is formulated and the asymptotical boundary conditions are obtained in Subsection 3.1. In Subsection 3.2 we combine the triangle expansion technique with the projection on the open channels method [15] and connect this approach with some kind of renormalization procedure.

Section 4 contains the application of the method under discussion to the exactly solvable model of the three-body one-dimensional system with point interaction. In Subsection 4.1 we reformulate this problem in terms of diffraction on a wedge. In Subsection 4.2 the asymptotical behaviour of connections operator $A$ is studied and its compactness in a suitable space is proved. Also the relations between the effective $S$-matrix $S^{\text {eff }}$ of the reduced problem and the global $S$-matrix of the total original problem are obtained there.

In Section 5 we consider the wave propagation by the curved quantum 
waveguidc (CQW) in the frames of the triangle expansion method under the condition of small curvature of CQW. We formulate there the inverse problem for CQW and suggest scheme for its solution.

\section{General SCheme of the Method}

\subsection{Notations and Formulation of the Problem}

We shall consider a quantum system whose dynamics is determined by the Hamiltonian $H$ acting in the Hilbert space $\mathfrak{H}$ represented in the form

$$
\mathfrak{S}=\int_{\mathscr{M}} \oplus \mathscr{F}_{x} d x
$$

where $\mathscr{M}$ is some manifold and $\widetilde{F}_{x}$ form the family of Hilbert spaces parametrized by the point $x \in \mathscr{M}$. Decomposition (1) is equivalent to the existence of a Hilbert bundle, where $\mathscr{M}$ is the base space, $\mathscr{F}_{x}$ are Hilbert fibres [16-18], $\mathfrak{R}$ is the total configuration space, i.e., $\mathfrak{G}$ is the Hilbert space of functions on $\mathfrak{R}$. We suppose the special structure of the Hamiltonian

$$
H=h \otimes I+\mathscr{L},
$$

where operator $\mathscr{L}$ can be represented in the form of a direct integral

$$
\mathscr{L}=\int_{\mathscr{M}} \oplus \mathscr{L}(x) d x
$$

and $\mathscr{L}(x)$ are the self-adjoint operators in the Hilbert fibres $\mathscr{F}_{x}$. In representation (2), $h \otimes I$ means the self-adjoint operator acting as $h$ on the variable $x \in \mathscr{M}$ and as identity operator on additional variables.

In this paper we are going to consider situations when $\mathfrak{S}=L^{2}(\mathscr{M} \times \mathscr{N}, d \hat{\mu}), d \hat{\mu}$ being some positive measure on $\mathscr{M} \times \mathscr{N}$. Then the Hilbert fibres are $\mathscr{F}_{x}=$ $L^{2}\left(\mathcal{N}, d m_{x}\right)$ and $h$ acts in the space $L^{2}(\mathscr{M}, d \mu)$, where $d \mu$ is the measure on $\mathscr{M}$ and $d m_{x}$ form the family of measures on $\mathscr{N}$, parametrized by the point $x \in \mathscr{M}$.

Let us consider the spectral problem for the Hamiltonian $H$ :

$$
H \Psi={ }_{z} \Psi
$$

In order to investigate this problem we are to use the method of so-called triangle expansions $[1,10-12]$ which means the transformation into the spectral representation for the operator $\mathscr{L}(x)$ :

$$
\Psi=\sum_{m} \int \chi_{m} \varphi_{m}
$$


Here $\varphi_{m}$ denote the eigenfunctions of the operator $\mathscr{L}(x)$ :

$$
\mathscr{L}(x) \varphi_{m}=\lambda_{m}(x) \varphi_{m}
$$

and the symbol $\sum_{m} \int$ stands for the summation over the discrete spectrum of the operator $\mathscr{L}(x)$ and integration over the continuous one. Due to the self-adjointness of $\mathscr{L}(x), \forall x \in \mathscr{M}$, the eigenfunctions $\left\{\varphi_{m}\right\}$ form the moving frame (i.e., the orthonormal basis which depends on the point $x \in \mathscr{M}$ prametrically) in the fibre $\mathscr{F}_{x}$. Hence, we call the operator $\mathscr{L}(x)$ at each fixed $x \in \mathscr{M}$ the frame Hamiltonian and the spectral problem (6) as the frame problem. In time-dependent problems, where $x=t$, the operator $\mathscr{L}(t)$ is called the instantaneous Hamiltonian.

The completeness of the set $\left\{\varphi_{m}\right\}$ provides the possibility to represent every $\Psi \in \mathfrak{H}$ in the form (5), which is called the triangle expansion. Coefficients $\chi_{m}=\chi_{m}(x)$ in the expansion (5) depend on the parameter $x \in \mathscr{M}$.

Let us substitute the representation (5) into Eq. (3) and project the result on $\varphi_{n}$ in the Hilbert fibre $\mathscr{F}_{x}$. Orthogonality of the set $\left\{\varphi_{m}\right\}$

$$
\left\langle\varphi_{m}, \varphi_{n}\right\rangle=\delta_{m n}, \quad \forall x \in \mathscr{M},
$$

leads to the following equation for the coefficents $\chi_{m}(x)$ :

$$
\sum \int_{m}\left\langle h \otimes I\left(\chi_{m} \varphi_{m}\right), \varphi_{n}\right\rangle \chi_{m}+\left(\lambda_{n}(x)-z\right) \chi_{n}(x)=0, \quad \forall n
$$

Here $\langle\cdot, \cdot\rangle$ means the inner product in $\mathscr{F}_{x}$.

Functions $\lambda_{n}(x)$ are the energy levels of the family of the frame Hamiltonians $\mathscr{L}(x)$. The main properties of the effective equation (7) are essentially determined by the fact of whether there exists level crossing or not (i.e., whether or not there exist such points $x_{0} \in \mathscr{M}$, in which $\lambda_{m}\left(x_{0}\right)=\lambda_{n}\left(x_{0}\right)$ for some $\left.m, n\right)$. The case of the absence of level crossing is essentially simpler, and in most concrete situations we suppose this condition to be valid.

Henceforth let us suppose the following structure of the operator $h$ :

$$
h=-\Delta_{x}+V(x)
$$

where $V(x)$ is a potential, or, more generally, an operator of interaction. This leads to the following version of the effective equation (7):

$$
\begin{gathered}
\sum \int_{m}-\left\langle\Delta_{x} \varphi_{m}, \varphi_{m}\right\rangle \chi_{m}-\left\langle\nabla_{x} \varphi_{m}, \varphi_{n}\right\rangle \nabla_{x} \chi_{m}-\Delta_{x} \chi_{n} \\
+\left(V(x)+\lambda_{n}(x)-z\right) \chi_{n}=0, \quad \forall n .
\end{gathered}
$$

Formal expression (9) evidently needs justification. Namely, one should be sure that the functions $\varphi_{m}$ have two derivatives over the parameter $x$. In every concrete situation we discuss this property. 
Let us rewrite the system (9) in a more convenient form. Namely, let us introduce the matrix $A(x)$ (infinite-dimensional in the general case) with the matrix elements

$$
A_{n m}(x)=\left\langle\nabla_{x} \varphi_{m}, \varphi_{n}\right\rangle .
$$

If $\varphi_{n}, \varphi_{m}$ are the wave functions of the continuous spectrum of the operator $\mathscr{L}(x)$, then $A(x)$ is the integral operator having the kernel $A_{n m}(x)$. In the general case the kernel $A_{n m}(x)$ is the distribution. Note that switching-on the regularizer (that transforms plane waves into wave packet) allows interpretation of the "continuous" part of $A(x)$ in the ordinary sense. The following lemma is valid.

LEMMA 1. If $\varphi_{m} \in C^{2}(\mathscr{M})$ for every $m$ and $\left\{\varphi_{m}\right\}$ is the orthonormul set for every $x \in \mathscr{M}$, the operator $A(x)$ defined by its matrix elements $A_{n m}(x)$ satisfies the equality

$$
\left\langle\Delta_{x} \varphi_{m}, \varphi_{n}\right\rangle=\left(\nabla_{x} A+A^{2}\right)_{n m} .
$$

Proof is based on direct calculations. Lemma 1 allows rewriting Eq. (9) in the form

$$
\left\{-\left(\nabla_{x} \otimes 1+A(x)\right)^{2}+A(x)+(V(x)-z) \otimes 1\right\} \chi(x)=0 .
$$

Here $A(x)=\operatorname{diag}\left\{\lambda_{n}(x)\right\}$ is the diagonal operator and 1 stands for the identical operator, acting over the "vector variables." "Vector coefficient $\chi=\left\{\chi_{m}(x)\right\}$ is the element of the space

$$
\mathscr{H}=\int_{\mathscr{M}} \oplus\left(\mathbb{C}^{N(x)} \oplus L^{2}(\mathfrak{M}, d v(\lambda)) d \mu(x),\right.
$$

where $L^{2}(\mathfrak{M}, d v(\lambda))$ is the Hilbert space, where spectral representation of the absolute continuous part of $\mathscr{L}(x)$ acts as the operator for multiplying by $f(x)$. Here $N(x)$ is the dimension of the discrete subspace of the Hamiltonian frame $\mathscr{L}(x)$, and $\sigma_{c}(x)$ is the continuous spectrum of $\mathscr{L}(x)$. Let us point out, that if $N(x)=\infty$ then $\mathbb{C}^{N(x)}=l_{2}$. The space $\mathscr{H}$ is $\mathscr{H}=L^{2}\left(\mathscr{M}, l_{2}, d \mu\right)$ if the $\mathscr{L}(x)$ have discrete spectrum only; this important case is considered in Section 4, 5. Here we only notice that in this case the triangle representation (5) can be written as

$$
\Psi=\left\langle\langle\chi, \bar{\varphi}\rangle_{l_{2}},\right.
$$

where $\varphi=\left\{\varphi_{m}\right\}$ and $\left\langle\langle\cdot, \cdot\rangle_{l_{2}}\right.$ stands for the inner product in $l_{2}$.

The effective equation $(12)$ is the principal object in the approach under discussion. The next two subsections are devoted to special interpretations of the operator $A(x)$, playing the main role in Eq. (12).

\subsection{Geometrical Interpretation}

The construction suggested in the previous subsection has a natural geometrical interpretation. The main goal of the corresponding geometrical considerations is to 
single out some important objects which determine the essential dynamical properties of the system. Objects usually discussed in quantum scattering theory, such as the resolvent, $T$-matrix, or $S$-matrix have a very complicated structure in the most interesting situations. In the frame of the approach under discussion we extract some invariant geometrical objects. The analysis of the remaining structures is sometimes a less difficult problem than analysis of the original problem.

The geometry in our construction starts from the Hilbert bundle corresponding to the representation (1) of the Hilbert space $\mathfrak{H}$. Under condition (8) the coefficients $A_{n m}(x)$ determine the connection 1-form $\mathscr{A}(x)$ on the Hilbert bundle described above. One can check this fact by considering the set $\left\{\varphi_{k}\right\}$ as the fibre vector $\varphi(\cdot, x), x \in \mathscr{M}$, and investigating the parallel transport of $\varphi(\cdot, x)$ when $x$ undergoes the shift $x \rightarrow x+d x$ :

$$
\varphi(\cdot, x+d x)=\varphi(\cdot, x)+\delta \varphi .
$$

Consider $x=\left\{x^{\mu}\right\} \in \mathscr{M}, \mu=1, \ldots, \operatorname{dim} \mathscr{M}$ and define vector components of the connection $\hat{A}^{\mu}$ as the coefficients in the representation

$$
\delta \varphi=\sum_{\mu} \hat{A}^{\mu}(x) d x^{\mu} \varphi
$$

On the other hand, one can represent $\delta \varphi$ in the form

$$
\delta \varphi=\sum_{\mu} \partial_{\mu} \varphi d x^{\mu},
$$

where $\partial_{\mu}=\partial / \partial x^{\mu}$. Writing the relations (14), (15) for the components

$$
\delta \varphi_{k}=\sum_{m} \int \sum_{\mu} \hat{A}_{k m}^{\mu} d x^{\mu} \varphi_{m} ; \quad \delta \varphi_{k}=\sum_{\mu} \partial_{\mu} \varphi_{k} d x^{\mu},
$$

one can see that

$$
\partial_{\mu} \varphi_{k}=\sum_{m} \int \hat{A}_{k m}^{\mu} \varphi_{m}
$$

The set $\left\{\varphi_{m}\right\}$ is the orthonormal basis; hence,

$$
\left\langle\partial_{\mu} \varphi_{s}, \varphi_{k}\right\rangle=\partial_{\mu}\left\langle\varphi_{s}, \varphi_{k}\right\rangle-\left\langle\varphi_{s}, \partial_{\mu} \varphi_{k}\right\rangle=-\overline{\left\langle\partial_{\mu} \varphi_{k}, \varphi_{s}\right\rangle},
$$

where the bar denotes a complex conjugation.

Projecting the relation (16) on $\varphi_{s}$ and using Eqs. (10) and (17), one can obtain the equation

$$
\begin{aligned}
A_{k s}^{\mu} & =\left\langle\partial_{\mu} \varphi_{s}, \varphi_{k}\right\rangle=-\overline{\left\langle\partial_{\mu} \varphi_{k}, \varphi_{s}\right\rangle} \\
& =-\sum_{m} \int \overline{\hat{A}_{k m}^{\mu}}\left\langle\varphi_{m}, \varphi_{s}\right\rangle=-\overline{\hat{A}_{k s}^{\mu}},
\end{aligned}
$$


where $\overline{\hat{A}^{\mu}}$ is a complex conjugated to $\hat{A}^{\mu}$. Thus $A(x)$ actually determines the connection $\hat{A}(x)$ :

$$
\hat{A}(x)=-\overline{A(x)}
$$

One can notice that the covariant derivative

$$
D_{\mu}=\partial_{\mu}+A^{\mu}(x)
$$

generated by connection $\hat{A}(x)$ allows rewriting Eq. (12) in the form

$$
\left\{-D^{2}(x)+\Lambda(x)+(V(x)-z)\right\} \chi(x)=0,
$$

where $D^{2}=\sum_{\mu} D_{\mu}^{2}$.

Let us construct the connection 1-form

$$
\mathscr{A}(x)=\sum_{\mu} \hat{A}^{\mu}(x) d x_{\mu}
$$

and define the 2-form of the curvature

$$
\mathfrak{F}=d \mathscr{A}+\mathscr{A} \wedge \mathscr{A}=\sum F^{\mu v} d x_{\mu} \wedge d x_{v}
$$

with the coefficients

$$
F^{\mu v}=\partial_{\mu} \hat{A}^{v}-\partial_{v} \hat{A}^{\mu}+\left[\hat{A}^{\mu}, \hat{A}^{v}\right] .
$$

The following lemma is valid.

Lemma 2. Under condition $\varphi(\cdot, x) \in C^{2}(\mathscr{M})$ the curvature

$$
F^{\mu v}=0 \text {. }
$$

The proof is based on relation $\partial_{\mu} \partial_{\nu}=\partial_{\nu} \partial_{\mu}$ for smooth $\varphi$ :

$$
\begin{aligned}
{\left[\hat{A}^{\mu}, \hat{A}^{v}\right]_{m n} } & =\sum_{k} \int\left\langle\varphi_{m}, \partial_{v} \varphi_{k}\right\rangle\left\langle\varphi_{k}, \partial_{\mu} \varphi_{n}\right\rangle-\left\langle\varphi_{m}, \partial_{\mu} \varphi_{k}\right\rangle\left\langle\varphi_{k}, \partial_{v} \varphi_{n}\right\rangle \\
& =\sum_{k} \int-\left\langle\partial_{v} \varphi_{m}, \varphi_{k}\right\rangle\left\langle\varphi_{k}, \partial_{\mu} \varphi_{n}\right\rangle+\left\langle\partial_{\mu} \varphi_{m}, \varphi_{k}\right\rangle\left\langle\varphi_{k}, \partial_{v} \varphi_{n}\right\rangle \\
& =-\left\langle\partial_{v} \varphi_{m}, \partial_{\mu} \varphi_{k}\right\rangle+\left\langle\partial_{\mu} \varphi_{k}, \partial_{v} \varphi_{n}\right\rangle .
\end{aligned}
$$

On the other hand,

$$
\begin{aligned}
& \left(\partial_{\mu} \hat{A}^{v}\right)_{m n}=\left\langle\partial_{v} \partial_{\mu} \varphi_{m}, \varphi_{n}\right\rangle+\left\langle\partial_{v} \varphi_{m}, \partial_{\mu} \varphi_{n}\right\rangle \\
& \left(\partial_{v} \hat{A}^{\mu}\right)_{m n}=\left\langle\partial_{\nu} \partial_{\mu} \varphi_{m}, \varphi_{n}\right\rangle+\left\langle\partial_{\mu} \varphi_{m}, \partial_{v} \varphi_{n}\right\rangle .
\end{aligned}
$$

Hence, using (23), we obtain (24). 
Thus we have demonstrated that the operator $A(x)$ generates the connection 1-form $\mathscr{A}(x)$. Before starting the study of $A(x)$ properties, let us point out that the infinite-dimensional character of $A(x)$, i.e., of the effective equation (12) introduces essential difficulties to the investigation and numerical solution. One of the possible ways to avoid this difficulty is to construct the fibre bundle with one-dimensional structure group $U(1)$, where each point $x \in \mathscr{M}$ of the base space $\mathscr{M}$ is connected with the single function. In principle it can be obtained in the frame of the operator quasiclassical approach [19]. We consider this problem elsewhere, and here we overcome the infinite-dimensional character of the effective equations in some other way. Namely, in model situations (see Sections 4,5 ) we prove the compactness of the operator $A(x)$ in suitable spaces, which gives an opportunity to approximate $A(x)$ by the finite-dimensional operators.

\subsection{Operator $A$ as a Gauge Field}

Let us consider the physical meaning of the procedure, described in Subsection 2.1. Using the triangle expansion (5) we have obtained the effective equation (12) instead of the original Schroedinger equation (4). One can notice that the "vector-coefficient" $\chi(x)$ determined by the effective equation (12) is a function defined on the manifold $\mathscr{M}$ which plays the role of the configuration space of a lower dimension in comparison with the configuration space of the original problem. Investigation of the effective scattering theory in the space of the lower dimension can be an easier problem than the analysis of the original many-dimensional problem, because in the approach under discussion the difficulties are partially transmitted to the frame problem (6). In this sense the triangle expansion technique can be treated as a version of dimensional reduction [10-12]. In many-body quantum scattering theory this procedure is associated with the possibility of clustering of particles and construction of an effective scattering theory for the clusters. In such a theory, from the quantum-mechanical point of view, clusters play the role of composite particles whose internal structure is displayed in the operator $A(x)$. On the other hand, one can interpret the vector $\chi=\left\{\chi_{m}\right\}$ as the infinitedimensional vector describing the material field. In this consideration the operator $A(x)$ plays the role of an effective gauge field mediating the interaction between quasi-particles .

Let us consider the relation between gauge transforms and geometrical construction described in a preceding subsection. We introduce the so-called evolution operator.

Consider a contour $C$ lying in the base space $\mathscr{M}$ and parameterized as follows: $C \ni x=x(\lambda) ; x_{0}=x(0)$. For the parallel transport of the fibre vector $\varphi(\cdot, x)$ along the contour $C$ (the adiabatic transport [16-18]) we have Eqs. (14), (18). Hence for $\varphi(\lambda)=\varphi(\cdot, x(\lambda))$ we obtain, after complex conjugation,

$$
\delta \bar{\varphi} / d \lambda+\sum_{\mu} A^{\mu}(x(\lambda)) d x^{\mu} / d \lambda \bar{\varphi}=0 .
$$


Equation (25), together with the initial condition $x\left(\lambda_{0}\right)=x_{0}$, generates the resolving operator $U\left(\lambda, \lambda_{0}\right)$ :

$$
\overline{\varphi(\lambda)}=U\left(\lambda, \lambda_{0}\right) \overline{\varphi\left(\lambda_{0}\right)}
$$

The set of the operators $U\left(\lambda, \lambda_{0}\right)$ satisfies the equation

$$
\begin{aligned}
d U\left(\lambda, \lambda_{0}\right) / d \lambda+\sum_{\mu} A^{\mu}(x(\lambda)) d x^{\mu} / d \lambda U\left(\lambda, \lambda_{0}\right) & =0, \\
U\left(\lambda_{0}, \lambda_{0}\right) & =I .
\end{aligned}
$$

The solution for problem (27) is given by the multiplicative integral

$$
U\left(\lambda, \lambda_{0}\right)=T \exp \left\{\int_{\lambda_{0}}^{\lambda} \sum_{\mu} A^{\mu}(x)\left(d x^{\mu} / d \lambda\right) d \lambda\right\}
$$

following the contour $C$ in $\mathscr{M}$. This $T$-exponent coincides with the well-known Wilson loop [6].

If the integral in Eq. (28) does not depend on the path (which is true if there is no level crossing for the frame Hamiltonian $\mathscr{L}(x)$ ), one can define the operatorvalued function $U(x)$ :

$$
U\left(\lambda, \lambda_{0}\right)=U\left(x(\lambda), x_{0}\right)=U(x) .
$$

We refer to the operator $U(x)$ acting from $\mathscr{F}_{x_{0}}$ to $\mathscr{F}_{x}$ and having the property (26) the evolution operator.

The condition of completeness of the set $\left\{\varphi_{k}\right\}$ in each fibre $\mathscr{F}_{x}$ can be written in the form

$$
U^{+}(x)=U^{-1}(x), \quad \forall x \in \mathscr{M} .
$$

Thus we have the relation between the evolution operator $U(x)$ and the effective gauge field $A(x)$. If the integral in Eq. (28) does not depend on the path, i.e., $U(x)$ can be defined, Eq. (27) leads to the equation

$$
\partial_{\mu} U+A^{\mu} U=0
$$

and, hence,

$$
A^{\mu}(x)=-\partial_{\mu} U(x) U^{-1}(x)
$$

Thus the covariant derivative associated with the gauge field $A^{\mu}$,

$$
D_{\mu}=\partial_{\mu}+A^{\mu}(x),
$$

can be rewritten in terms of the operator $U(x)$ :

$$
D_{\mu}=\partial_{\mu}-\partial_{\mu} U U^{-1} \text {. }
$$


Under condition (30) the gaugc ficld $A(x)$ can be removed by the corresponding gauge transform $\chi^{\prime}=U^{-1} \chi$.

In order to obtain this fact we need the following:

LEMMA 3. If $U^{-1}=U^{+}$, then

$$
U \partial_{\mu}^{2} U^{-1}=\left(\partial_{\mu}-\partial_{\mu} U U^{-1}\right)^{2} .
$$

Proof of this lemma is based on the relation

$$
\partial_{\mu} U^{-1}=-U^{-1}\left(\partial_{\mu} U\right) U^{-1} \text {. }
$$

Relation (32) and Lemma 3 allow to rewriting Eq. (12) in the form

$$
\left\{-U \Delta_{x} \circ U^{-1}+A U U^{-1}-E U U^{-1}\right\} \chi=0 .
$$

Using the gauge transform

$$
\chi^{\prime}(x)=U^{-1}(x) \chi(x),
$$

we obtain the following equation for $\chi^{\prime}(x)$,

containing no gauge field.

$$
\left\{-\Delta_{x}+U^{-1} \Lambda U-E\right\} \chi^{\prime}=0
$$

The anti-Hermitian operator $A(x)$ can also be interpreted as the operator of nonadiabacity. Indeed, the off-diagonal elements $A_{m n}(x), m \neq n$, determine the coupling of equations in system (12). Adiabatic behaviour of the system, i.e., trivial evolution of eigenfunction $\varphi_{n}(x): \varphi_{n}(x)=\exp \left(i \delta\left(x, x_{0}\right)\right) \varphi_{n}\left(x_{0}\right)$ implies the vanishing of diagonal terms: $A_{m m}(x) \equiv 0$.

Let us notice that if eigenfunction $\varphi_{n}$ can be chosen real globally, the diagonal elements of the operator $A(x)$ are equal to zero: $A_{n n}(x)=0, \forall n$. However, in the general case, this choice of gauge is not always possible globally [20].

If we locally change the choice of phases for $\varphi_{n}(x)$ :

$$
\begin{gathered}
\mathscr{U}(x):\left\{\varphi_{n}(x)\right\} \mapsto\left\{\exp \left(i \xi_{n}(x)\right) \varphi_{n}(x)\right\}, \\
\mathscr{U}(x)=\operatorname{diag}\left\{\exp \left(i \xi_{n}(x)\right\}, \quad \xi_{n}(x) \in R,\right.
\end{gathered}
$$

the effective gauge field $A(x)$ undergoes the transform

$$
\hat{\mathscr{U}}(x): A(x) \mapsto \mathscr{U}^{-1} A \mathscr{U}+\nabla_{x} \mathscr{U} \mathscr{U}^{-1} .
$$

\section{Three-Body System in Triangle Representation}

\subsection{Representation of the Three-Body Hamiltonian}

In this section we consider the application of the general scheme described above to the system of three quantum spinless particles in $\mathbb{R}^{3}$. 
Let us consider a system of threc spinless quantum particles whose reduced configuration space is treated as a manifold having atlas $\left\{\mathscr{X}_{\alpha}\right\}$ consisting of three maps $\mathscr{X}_{\alpha}, \alpha=1,2,3$. Each $\mathscr{X}_{x}$ corresponds to one extracted set of Jacobi coordinates $X=\left\{x_{\alpha}, y_{\alpha}\right\}$. There exists a $C^{\infty}$-diffeomorphism between $\mathscr{X}_{\alpha}$ and $\mathbb{R}^{6}$, and in the local coordinates $X=\left\{x_{\alpha}, y_{\alpha}\right\}$ in the map $\mathscr{X}_{\alpha}$, the space $\mathbb{B}^{6}$ can be represented in the form $\mathbb{R}^{6}=\mathbb{R}_{x_{\alpha}}^{3} \times \mathbb{R}_{v_{\alpha}}^{3}$. The local coordinates $\left\{x_{\alpha}, y_{x}\right\}$ for different $\alpha$ are connected by orthogonal matrices which are parametrized by the masses of the particles [21].

The Hamiltonian of the system acts in the Hilbert space $\mathfrak{H}=L^{2}\left(\mathbb{R}^{6}\right)$ and can be represented in the form

$$
\begin{aligned}
H & =-\Delta_{X}+\sum_{\beta} V_{\beta}\left(x_{\beta}\right) \\
& =\left(-\Delta_{y_{\alpha}}\right) \otimes I_{x_{x}}+I_{y_{x}} \otimes\left(-\Delta_{x_{\alpha}}\right)+\sum_{\beta} V_{\beta}\left(x_{\beta}\right),
\end{aligned}
$$

where $V_{\beta}\left(x_{\beta}\right)$ are the interactions between the particles. This operator is the selfadjoint on the domain $\mathscr{D}(H)=\mathscr{D}\left(-\Delta_{X}\right) \subset \mathfrak{H}$.

There are several versions of the application of the general scheme described in Section 2 to this problem. The choice of the base space determines which coordinate serves as the effective dynamical variable and which coordinate stands for the parameter. If one used $\mathbb{R}_{x_{\alpha}}^{3}$ as the base space he would obtain the effective twocenter problem at each fixed $x_{\alpha}$. Another possibility is given by the choice of the base space $\mathscr{M}=\mathbb{R}_{y_{x}}^{3}$ which is used in the paper. On the formal level there is no difference between the general scheme of the application of the triangle expansions method in these two cases; however, the version $\mathscr{M}=\mathbb{R}_{y_{x}}^{3}$ can be easily generalized for the many-body systems with natural two-cluster clusterisation, such as $d+t$ or $d+\alpha$ systems at the energies less than $\alpha$-particle break-up threshold [10].

Another version of the triangle expansion technique for the three-body system is given by the so-called hyperspherical adiabatic method in total angular momentum representation, where the half-axis $\mathbb{E}_{r}^{+}$serves as the base space $(r$ stands for hyperradius). We consider such an approach in Section 4 in the one-dimensional model.

Thus we consider the Hilbert space $\mathfrak{H}=L^{2}\left(\mathbb{R}^{6}\right)$ and the fibre bundle over the base space $L^{2}\left(\mathbb{R}_{y_{x}}^{3}\right)$ with Hilbert fibres $\mathscr{F}_{y_{x}}=L^{2}\left(\mathbb{R}_{x_{x}}^{3}\right)$.

We have some freedom in the representation of the Hamiltonian (42) in the form of (2), where the operator $h$ has the form (8). Sometimes it is useful to extract some effective interaction $V^{\text {eff }}\left(y_{\alpha}\right)[10]$,

$$
\sum_{\beta} V_{\beta}\left(x_{\beta}\right)=V^{\mathrm{eff}}\left(y_{\alpha}\right)+\hat{V}\left(x_{\alpha}, y_{\alpha}\right),
$$

and represent the operator $H$ in the form

$$
H=\left[-\Delta_{y_{\alpha}}+V^{\mathrm{eff}}\left(y_{x}\right)\right] \otimes I_{x_{x}}+\int \oplus \hat{\mathscr{L}}\left(y_{\alpha}\right) d y_{\alpha},
$$

where $\hat{\mathscr{L}}\left(y_{\alpha}\right)=-\Delta_{x_{\alpha}}+\hat{V}\left(x_{\alpha}, y_{\alpha}\right)$. 
Here we represent the Hamiltonian $H$ in the form

$$
H=\left(-\Delta_{v_{x}}\right) \otimes I+\mathscr{L},
$$

where

$$
\mathscr{L}=I \otimes\left(-A_{x_{x}}\right)+\sum_{\beta} V_{\beta}=\int_{\mathbb{R}^{3}} \oplus \mathscr{L}\left(y_{x}\right) d y_{x} .
$$

The following assertion is obviously true.

LeMma 4. If $V\left(x_{\beta}\right) \rightarrow 0$ as $x_{\beta} \rightarrow \infty$, then the operator $\mathscr{L}\left(y_{\alpha}\right)=-\Delta_{x_{\alpha}}+\sum V_{\beta}$ is self-adjoint on the domain $\mathscr{D}\left(\mathscr{L}\left(y_{\alpha}\right)\right)=\mathscr{D}\left(-\Delta_{X_{x}}\right) \subset \mathscr{F}_{y_{2}}$. The discrete spectrum of the operator $\mathscr{L}\left(y_{\alpha}\right), \sigma_{d}\left(\mathscr{L}\left(y_{\alpha}\right)\right)=\left\{\varepsilon \lambda_{k}\left(y_{x}\right)\right\}$ determines the levels $\lambda_{k}\left(y_{\alpha}\right)$; the continuous spectrum $\sigma_{c}\left(\mathscr{L}\left(y_{\alpha}\right)\right)=[0, \infty)$, for every $y_{x}$.

Lemma 4 ensures the self-adjointness of the operator $\mathscr{L}\left(y_{\alpha}\right)$ in the space $\mathscr{F}_{y_{x}}$ and thus allows application of the general scheme described in Section 2, i.e., representing the solution $\Psi(X)$ of the eigenvalue problem (4) in the form of the triangle expansion (5), which looks like

$$
\Psi(X)=\sum_{k} \int \chi_{k}\left(y_{\alpha}\right) \varphi_{k}\left(x_{x}, y_{\alpha}\right)
$$

in the situation under discussion.

The effective equation (12) turns into

$$
\left\{-\left(\nabla_{y_{x}}+A\left(y_{\alpha}\right)\right)^{2}+\Lambda\left(y_{\alpha}\right)-z\right\} \chi\left(y_{\alpha}\right)=0,
$$

where $A\left(y_{\alpha}\right)=\operatorname{diag}\left\{\varepsilon_{k}\left(y_{x}\right)\right\}, A\left(y_{x}\right)=\left\{A_{k m}\left(y_{x}\right)\right\}$,

$$
A_{k m}\left(y_{x}\right)=\left\langle\nabla_{y_{x}} \varphi_{m}\left(x_{x}, y_{x}\right), \varphi_{k}\left(x_{\alpha}, y_{x}\right)\right\rangle .
$$

Let us notice that for the potentials $V_{\alpha}$, analytical under dilations, functions $\varphi_{m}$ have two derivatives over the parameter $y_{x}^{4}[4]$, and thus the operator $\nabla_{y_{x}}$ in Eq. (46) has the standard meaning.

Equation (46) determines the effective dynamics on the non-compact base space $\mathscr{M}=\mathbb{R}_{y_{\alpha}}^{3}$; thus in order to obtain the unique solution of Eq. (46), one should add the asymptotical boundary conditions for $\chi_{m}\left(y_{\alpha}\right)$ at $y_{\alpha} \rightarrow \infty$ to Eq. (46). Coefficients $\chi_{m}\left(y_{\alpha}\right)$ in the triangle expansion (45) are

$$
\chi_{m}\left(y_{x}\right)=\left\langle\Psi, \varphi_{m}\left(x_{x}, y_{\alpha}\right)\right\rangle .
$$

Equation (48) demonstrates that the asymptotics of $\chi_{m}\left(y_{\alpha}\right)$ is determined by the asymptotics of functions $\Psi$ and $\varphi_{m}$. The asymptotical behaviour of the three-body wave function has been studied in detail in [21], thus the problem turns into the problem of moving frame $\left\{\varphi_{m}\right\}$ asymptotics construction. 
Let us start from consideration of the discrete spectrum eigenfunctions of $\mathscr{L}\left(y_{\alpha}\right)$, determined by the equation

$$
\mathscr{L}\left(y_{\alpha}\right) \varphi_{n}\left(x_{\alpha}, y_{\alpha}\right)=\varepsilon_{n}\left(y_{\alpha}\right) \varphi_{n}\left(x_{\alpha}, y_{\alpha}\right) .
$$

The following statement is valid.

Lemma 5. If $v_{\gamma}\left(x_{\gamma}\right) \sim\left|x_{\gamma}\right|^{-3-v}, v>0$, at $\left|x_{\gamma}\right| \rightarrow \infty$, then $\varphi_{n}$ has the asymptotical representation

$$
\varphi_{n}\left(x_{\alpha}, y_{\alpha}\right)=Q_{\alpha}^{n}+\sum_{\beta \neq \alpha} Q_{\beta}^{n}
$$

where

$$
\begin{aligned}
Q_{\alpha}^{n}\left(x_{\alpha}, y_{\alpha}\right) & =\left(\Delta_{x_{\alpha}}+\varepsilon_{n}\left(y_{\alpha}\right)\right)^{-1} v_{x} \varphi_{n} \underset{\left|x_{x}\right| \rightarrow \infty}{=} \\
& =f_{\alpha}^{n}\left(\hat{x}_{\alpha}, y_{\alpha}\right) \exp \left(-\sqrt{\varepsilon_{n}\left(y_{\alpha}\right)}\left|x_{\alpha}\right|\right)\left|x_{\alpha}\right|^{-1}+O\left(\left|x_{\alpha}\right|^{-2}\right), \\
Q_{\beta}^{n}\left(x_{\alpha}, y_{\alpha}\right) & =\left(\Delta_{x_{x}}+\varepsilon_{n}\left(y_{\alpha}\right)\right)^{-1} v_{\beta} \varphi_{n}= \\
& =f_{\beta}^{n}\left(\hat{x}_{\beta}, y_{\beta}\right) \exp \left(-\sqrt{\varepsilon_{n}\left(y_{\alpha}\right)}\left|x_{\beta}\right| /\left|c_{\beta \alpha}\right|\right)\left|x_{\beta}\right|^{-1}+O\left(\left|x_{\beta}\right|^{-2}\right) .
\end{aligned}
$$

Here $f_{\gamma}^{n}$ are some smooth functions, $x_{\beta}=c_{\beta \alpha} x_{\alpha}+s_{\beta \alpha} y_{\alpha}, y_{\beta}=-s_{\beta \alpha} x_{\alpha}+c_{\beta \alpha} y_{\alpha}$, and $\hat{x}_{\alpha}=x_{\alpha} /\left|x_{\alpha}\right|$.

This lemma can be proved by methods of integral equations [36]. Namely, one can rewrite (49) in the integral form

$$
\varphi_{n}\left(x_{\alpha}, y_{\alpha}\right)=-\sum_{\beta} \int d x_{\alpha} \frac{\exp \left\{-\sqrt{\left|\varepsilon_{n}\left(y_{\alpha}\right)\right|}\left|x_{\alpha}-x_{\alpha}^{\prime}\right|\right\}}{4 \pi\left|x_{\alpha}-x_{\alpha}^{\prime}\right|} v_{\beta}\left(x_{\beta}^{\prime}\right) \varphi_{n}\left(x_{\alpha}^{\prime}, y_{\alpha}\right)
$$

and calculate the asymptotics of the right-hand side components:

$$
Q_{\beta}^{n}=-\int d x_{\alpha}^{\prime} \frac{\exp \left\{-\sqrt{\left|\varepsilon_{n}\left(y_{\alpha}\right)\right|}\left|x_{\alpha}-x_{\alpha}^{\prime}\right|\right\}}{4 \pi\left|x_{\alpha}-x_{\alpha}^{\prime}\right|} v_{\beta}\left(x_{\beta}^{\prime}\right) \varphi_{n}\left(x_{\alpha}^{\prime}, y_{\alpha}\right) .
$$

The result is given by Eqs. (50)-(52). We can obtain more detailed information about asymptotical behaviour of the basis $\varphi$. Namely, let us denote as $\sigma_{d}\left(h_{\alpha \gamma}\right)$ the discrete spectrum of the two-body Hamiltonians

$$
h_{\alpha \gamma}=-\Delta_{x_{\gamma}}+c_{\gamma \alpha}^{2} v_{\gamma}\left(x_{\gamma}\right)
$$

and give the following definition. We say that there is no accidental degeneracy in the system if

$$
c_{\beta \alpha}^{2} \sigma_{d}\left(h_{\alpha \beta}\right) \cap c_{\gamma \alpha}^{2} \sigma_{d}\left(h_{\alpha \gamma}\right)=\varnothing
$$

for every $\beta, \gamma=1,2,3\left(c_{x x}=1\right)$. 
LEMMA 6. If for every $\gamma=1,2,3$,

(1) $v_{y} \in L_{2}\left(\mathbb{R}^{3}\right)$;

(2) $v_{\gamma}\left(x_{\gamma}\right)$ is continuous for sufficiently large $x_{\gamma}$;

(3) there is no accidental degeneracy in the system, then

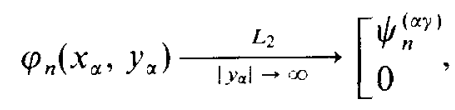

for some $\gamma=1,2,3$, where $\psi_{n}^{(\alpha \gamma)}$ is an eigenfunction of the Hamiltonian $h_{\alpha \gamma}$.

The proof of this statement can be given by rewriting Eq. (49) in form of the Faddeev equations and estimating the $L_{2}$-norm of the right-hand side of these equations at $\left|y_{\alpha}\right| \rightarrow \infty$.

For the continuous spectrum wave functions $\varphi_{4}$,

$$
\mathscr{L}\left(y_{\alpha}\right) \varphi_{q}=q^{2} \varphi_{q}, \quad q^{2}>0,
$$

we have a result similar to Lemma 5.

LEMMa 7. Under conditions of Lemma 5 the following asymptotics are valid:

$$
\begin{aligned}
\varphi_{q}\left(x_{\alpha}, y_{\alpha}\right) & =\exp \left\{i\left\langle x_{x}, q\right\rangle\right\}+Q_{\alpha}^{q}+\sum_{\beta \neq \alpha} Q_{\beta}^{q}, \\
Q_{\alpha}^{q}\left(x_{\alpha}, y_{\alpha}\right) & =\left(\Delta_{x_{\alpha}}+q^{2}+i 0\right)^{-1} v_{x} \varphi_{q} \underset{\left|x_{\alpha}\right| \rightarrow \infty}{=} \\
& =f_{\alpha}^{q}\left(\hat{x}_{\alpha}, y_{x}\right) \exp \left\{i|q|\left|x_{\alpha}\right|\right\}\left|x_{\alpha}\right|^{-1}+O\left(\left|x_{\alpha}\right|^{-2}\right), \\
Q_{\beta}^{q}\left(x_{\alpha}, y_{x}\right) & =\left(\Delta_{x_{x}}+q^{2}\right)^{-1} v_{\beta} \varphi_{q_{\mid x \beta} \mid \rightarrow \infty}= \\
& =f_{\beta}^{q}\left(\hat{x}_{\beta}, y_{\beta}\right) \exp \left\{i|q|\left|x_{\alpha}\right| /\left|c_{\beta \alpha}\right|\right\}\left|x_{\beta}\right|^{-1}+O\left(\left|x_{\beta}\right|^{-2}\right),
\end{aligned}
$$

Here $f_{\gamma}^{q}$ are some smooth functions (the amplitudes of appropriate processes).

The proof can be given by the same methods [36] as for Lemma 5 .

\subsection{Projecting on Open Channels and Renormalization}

Now we are to connect the spectral decomposition of the operators $A$ and $U$ defined in Section 2 with some kind of renormalization procedure. In order to do that let us rewrite Eq. (46) in another form,

$$
\left\{-D^{2}+a^{+} a+\langle V\rangle\right\} \chi=z \chi,
$$

where $D^{2}=\sum_{\mu} D_{\mu}^{2}, a^{+} a$ is a non-negative operator which can be interpreted as the mass operator and is determined by the matrix elements

$$
\begin{gathered}
\left(a^{+}\right)_{k m}=\left\langle\nabla_{x_{x}} \varphi_{m}, \varphi_{k}\right\rangle, \\
\left(a^{+} a\right)_{k m}=-\left\langle\Delta_{x_{x}} \varphi_{m}, \varphi_{k}\right\rangle,
\end{gathered}
$$


and

$$
\langle V\rangle=\left\{V_{k m}\right\}, \quad V_{k m}=\left\langle V \varphi_{m}, \varphi_{k}\right\rangle, \quad V=\sum_{\beta} V_{\beta} .
$$

As it was shown in Section 2, for smooth operators $U$, the gauge field $A^{\mu}=-\partial_{\mu} U U^{-1}$ has the trivial curvature $F^{\mu v}=0$ (see Lemma 2). The non-trivial part of the conncetion $\hat{A}=-\bar{A}$ can bc expressed in tcrms of the spectral projections $P^{d, c}\left(y_{\alpha}\right)$ on the discrete and continuous subspaces of the operator $\mathscr{L}\left(y_{x}\right)$ :

$$
\begin{aligned}
A^{\mu} & =-\partial_{\mu} U U^{-1}=-\partial_{\mu}\left[\left(P^{d}+P^{c}\right) U\right]\left[\left(P^{d}+P^{c}\right) U\right]^{+} \\
& =-\partial_{\mu}\left(P^{d} U\right)\left(P^{d} U\right)^{+}+M=A_{d}^{\mu}+M,
\end{aligned}
$$

where

$$
\begin{aligned}
M & =-\partial_{\mu}\left[\left(P^{d}+P^{c}\right) U\right]\left(P^{c}\right)^{+}-\partial_{\mu}\left(P^{c} U\right)\left[\left(P^{d}+P^{c}\right) U\right]^{+}, \\
A_{d}^{\mu} & =-\partial_{\mu}\left(P^{d} U\right)\left(P^{d} U\right)^{+} .
\end{aligned}
$$

The curvature $F_{d}^{\mu v}$ associated with the gauge field $A_{d}^{\mu}$ is non-trivial $\left(F^{\mu v} \neq 0\right)$ iff the restriction $P_{y_{\alpha}}^{d} U\left(y_{\alpha}\right)\left\ulcorner P_{y_{\alpha}}^{d} \mathscr{F}_{y_{\alpha}}\right.$ is not unitary $[1,12]$. Thus extracting the non-trivial part $A_{d}^{\mu}$ of the gauge field in the Fq. (34) is equivalent to redefining the covariant derivative $D \rightarrow D_{d}=\nabla_{y_{x}}+A_{d}^{\mu}$ and simultaneous renormalization of the mass operator:

$$
a^{+} a \rightarrow a^{+} a+M .
$$

However, the method mentioned above is a special case of a more general scheme, which is based on the projection on the open channels $[10,12,15]$.

Let us define the projection operators

$$
\mathbf{P}=\sum_{k \text {-open }} \int\left\langle\cdot, \varphi_{k}\right\rangle \varphi_{k}, \quad \mathbf{Q}=I-\mathbf{P}
$$

in the space $\mathfrak{H}$. Here the symbol $\sum_{k \text {-open }} \int$ stands for the summation and integration over the part of the spectrum of the operator $\mathscr{L}\left(y_{\alpha}\right)$ only;

$$
\sigma\left(\mathscr{L}\left(y_{\alpha}\right)\right) \ni \lambda_{k} \leqslant E_{0},
$$

where $E_{0}$ is some fixed energy. We refer to the subspace $\mathbf{P} \mathfrak{Y}$ as the open channels at energy $E_{0}$, and $\mathbf{Q S}$ as the closed channels. One can represent the wave function $\Psi$ in the form $\Psi=\mathbf{P} \Psi+\mathbf{Q} \Psi$ and use the triangle representation in the open channels only;

$$
\Psi=\mathbf{P} \Psi+\mathbf{Q} \Psi=\sum_{k \text {-open }}\left\langle\Psi, \varphi_{k}\right\rangle \varphi_{k}+\mathbf{Q} \Psi
$$

where we sum over indices $k$ corresponding to the open channels only. 
Eliminating the term $\mathbf{Q} \Psi$ from Schroedinger equation (4), one can obtain the effective equation for $\chi_{\mathbf{P}}=\left\{\chi_{k}\right\}_{k \text {-open }}=\left\{\left\langle\Psi, \varphi_{k}\right\rangle\right\}$, analogous to Eq. (46):

$$
\left\{-\left(\nabla_{y_{\alpha}}+A_{\mathbf{P}}\right)^{2}+A_{\mathbf{P}}\left(y_{x}\right)+A_{\mathbf{Q}}^{2}+W(z)-z\right\} \chi_{\mathbf{P}}=0 .
$$

Here $A_{\mathbf{P}}=\left\{A_{k m}\right\}_{k, m \text {-open }}=\left\{\left\langle\nabla_{y_{x}} \varphi_{m}, \varphi_{k}\right\rangle\right\}_{k, m \text {-open }}$ is the gauge field on the open channels,

$$
\begin{gathered}
A_{\mathbf{Q}}^{2}=\left\{A_{\mathbf{Q} k m}^{2}\right\}=\left\{\left\langle\mathbf{Q} \nabla_{y_{x}} \varphi_{m}, \mathbf{Q} \nabla_{y_{x}} \varphi_{k}\right\rangle\right\}, \\
A_{\mathbf{P}}=\operatorname{diag}\left\{\lambda_{k}\left(y_{\alpha}\right)\right\}_{k-\text { open }}, \\
W(z)=\left\{W_{i j}(z)\right\} ; \\
W_{i j}(z): \chi_{j} \mapsto-\left\langle H \mathbf{Q}(\mathbf{Q} H \mathbf{Q}-z)^{-1} \mathbf{Q} H\left(\varphi_{j} \chi_{j}\right), \varphi_{i}\right\rangle .
\end{gathered}
$$

Equation (65) shows that the effect of closed channcls is displaycd in the redefinition of the covariant derivative $D \rightarrow D_{\mathbf{P}}=\nabla_{y_{\alpha}}+A_{\mathbf{P}}$ and the corresponding renormalization of the mass operator, $a^{+} a \rightarrow a_{\mathbf{P}}^{+} a_{\mathbf{P}}+A_{\mathbf{Q}}^{2}+M_{\mathbf{P}}$, where

$$
\begin{aligned}
\left(a_{\mathbf{P}}^{+}\right)_{k m} & =\left\langle\nabla_{x_{\alpha}} \varphi_{m}, \varphi_{k}\right\rangle_{k, m-\text { open }}, \\
\left(M_{\mathbf{P}}\right)_{k m} & =\left\langle\mathbf{Q} \nabla_{y_{x}} \varphi_{m}, \mathbf{Q} \nabla_{y_{x}} \varphi_{k}\right\rangle_{k, m-\text { open }} .
\end{aligned}
$$

In addition, the closed channels make a contribution to Eq. (65) in the form of energy-dependent non-local operator $W(z)$. Let us define

$$
\begin{aligned}
\mathscr{A}_{p}\left(y_{\alpha}\right) & =\sum_{\mu=1}^{3} \hat{A}_{p}^{\mu}\left(y_{\alpha}\right) d y_{\alpha}^{\mu} \\
\hat{A}_{p}^{\mu} & =\left\{\left(\hat{A}_{p}^{\mu}\right)_{m n}\right\}_{m, n-\mathrm{open}}, \quad\left(\hat{A}_{p}^{\mu}\right)_{m n}=A_{n m}^{\mu}=-\overline{A_{n m}^{\mu}}
\end{aligned}
$$

and corresponding curvature 2-form,

$$
\mathscr{F}_{p}=\sum_{\mu<\nu} F_{p}^{\mu \nu} d y_{\alpha}^{\mu} \wedge d y_{\alpha}^{v}
$$

where

$$
F_{p}^{\mu v}=\partial_{\mu} \hat{A}_{p}^{v}-\partial_{v} \hat{A}_{p}^{\mu}+\left[\hat{A}_{p}^{\mu}, \hat{A}_{p}^{v}\right] .
$$

We call the subspace $\mathscr{F}_{0} \subset \mathscr{F}=L_{2}\left(\mathbb{R}_{x_{x}}^{3}\right)$ the adiabatic subspace for the set of the frame operators $\left\{L\left(y_{\alpha}\right)\right\}$ in the region $\mathscr{M}_{0} \subset \mathscr{M}=\mathbb{R}_{y_{x}}^{3}$, if for every $\varphi_{n}\left(\xi_{0}\right) \in \mathscr{F}_{0}$ (at some $\left.\xi_{0} \in \mathscr{M}_{0}\right)$, the following statement is valid:

$$
\varphi_{n}(\xi) \in \mathscr{M}_{0} \quad \text { for every } \xi \in \mathscr{M}_{0} .
$$

LEMMA 8. If $\varphi\left(y_{\alpha}\right) \in C^{2}\left(\mathbb{R}_{y_{x}}^{3}\right)$ and the subspace $\mathscr{F}_{0}=\mathbb{V}_{n \text {-open }}\left\{\varphi_{n}\right\}$ is the adiabatic subspace for the set $\left\{L\left(y_{\alpha}\right)\right\}$ in the region $\mathscr{M}_{0} \subset \mathbb{R}_{y_{\alpha}}^{3}$, then the corresponding curvature is equal to zero on $\mathscr{M}_{0}$ :

$$
\left.F_{p}\right|_{\mu_{0}} \equiv 0
$$


Proof. Using the relations obtained in the proof of Lemma 2, we have

$$
\left(F_{p}^{\mu \nu}\right)_{m n}=\sum_{s-c l o s e d} \int\left(\left\langle\partial_{\mu} \varphi_{m}, \varphi_{s}\right\rangle\left\langle\varphi_{s}, \partial_{\nu} \varphi_{n}\right\rangle-\left\langle\partial_{\nu} \varphi_{m}, \varphi_{s}\right\rangle\left\langle\varphi_{s}, \partial_{\mu} \varphi_{n}\right\rangle\right)
$$

If $\mathscr{F}_{0}$ is the adiabatic subspace for $\left\{L\left(y_{x}\right)\right\}$ in $\mathscr{M}_{0}$, then for every $\varphi_{n} \in \mathscr{F}_{0}$ (i.e., $n$-open), $\forall y \in \mathscr{M}_{0}$, we have

$$
\varphi_{n}\left(y_{\alpha}+d y_{\alpha}\right) \in \mathscr{F}_{0}
$$

On the other hand, for smooth $\varphi_{n}$,

$$
\varphi_{n}\left(y_{\alpha}+d y_{\alpha}\right)=\varphi_{n}\left(y_{\alpha}\right)+\sum_{\mu} \partial_{\mu} \varphi_{n} d y_{\alpha}^{\mu} .
$$

Hence $\partial_{\mu} \varphi_{n} \in \mathscr{F}_{0}$, and, consequently, $\left\langle\partial_{\mu} \varphi_{n}, \varphi_{s}\right\rangle=0$ for every $s$-closed. Using this fact and Eq. (71) we obtain (70).

Thus, Lemma 8 allows formulation of the necessary condition of the adiabatic property in the form of the zero-curvature equation:

$$
d \mathscr{A}_{p}=\frac{1}{2}\left[\hat{\mathscr{A}}_{p}, \hat{\mathscr{A}}_{p}\right]
$$

\section{Three-Body Scattering in ONe Dimension}

\subsection{Formulation of the Problem: Frame Hamiltonian}

We consider the quantum system of three spinless particles interacting with deltafunction potentials of equal strength $g \in \mathbb{R}$ in one dimension $[22-26,30]$. We suppose the wave function of the system to be symmetric; i.e., consider the statistics of boson particles. In the c.m. frame, the scattering in such a system is equivalent [22-26] to that in the sector $\Phi \in \mathbb{R}^{2}$ (see Fig. 1):

$$
\Phi=\left\{(r, \phi): r \in \mathbb{R}_{+},-\pi / 6 \leqslant \phi \leqslant \pi / 6\right\} .
$$

In polar coordinates $(r, \phi)$ the Hamiltonian of the system acts in the Hilbert space $L^{2}\left(\mathbb{R}^{+} x[-\pi / 6, \pi / 6], r d r d \phi\right)$ and has the form

$$
\begin{gathered}
H=-\left(\frac{\partial^{2}}{\partial r^{2}}+\frac{1}{r} \frac{\partial}{\partial r}+\frac{1}{r^{2}} \frac{\partial^{2}}{\partial \phi^{2}}\right) \\
\operatorname{Dom}(H)=L^{2}(\Phi, r d r d \phi) \cap\left\{u:-\left.\frac{1}{r} \frac{\partial u}{\partial \phi}\right|_{\phi= \pm \pi / 6}= \pm\left. g u\right|_{\phi= \pm \pi / 6}\right\} .
\end{gathered}
$$

Condition $\left.u\right|_{r=0}<\infty$ means the absence of three-body forces in the system [26].

It is convenient to use the standard change of variables

$$
v(r, \phi)=\sqrt{r} u(r, \phi),
$$




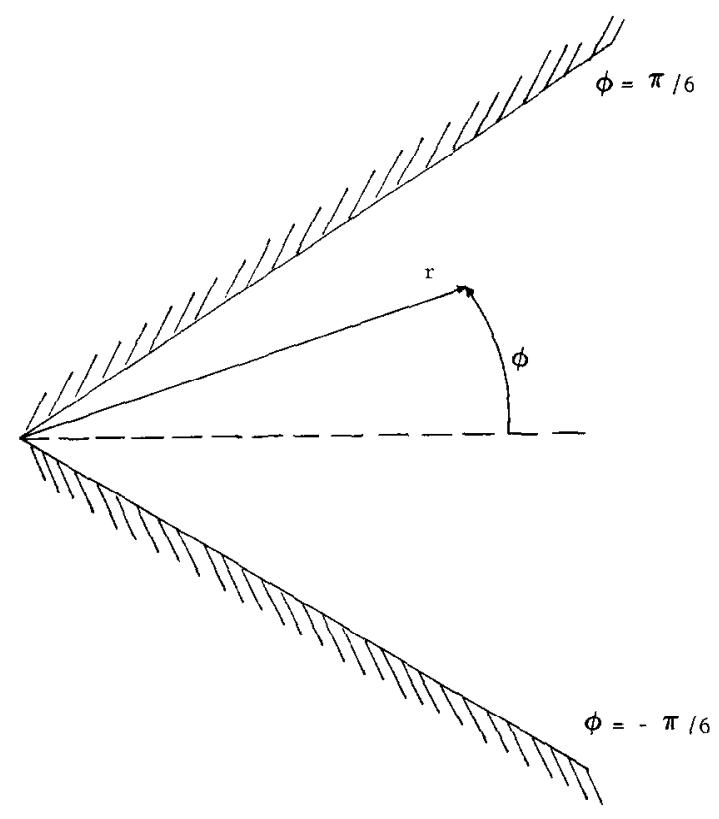

FIG. 1. The sector $\Phi$.

which leads to the spectral problem in the space $\mathfrak{S}=L^{2}\left(\mathbb{R}_{+} \times[-\pi / 6, \pi / 6], d r d \phi\right)$,

$$
\tilde{H} v=z v,
$$

where

$$
\begin{gathered}
\tilde{H}=-\left(\frac{\partial^{2}}{\partial r^{2}}+\frac{1}{4 r^{2}}+\frac{1}{r^{2}} \frac{\partial^{2}}{\partial \phi^{2}}\right) \\
\operatorname{Dom}(\tilde{H})=\mathfrak{H} \cap\left\{v:-\left.\frac{1}{r} \frac{\partial v}{\partial \phi}\right|_{\phi= \pm \pi / 6}= \pm\left. g v\right|_{\phi= \pm \pi / 6}\right\} .
\end{gathered}
$$

The spectral problem (75) is equivalent to the original spectral problem

$$
H u=z u \text {. }
$$

The operator $\tilde{H}$ can be represented in the form (2):

$$
\begin{aligned}
& \tilde{H}=-\partial_{r}^{2} \otimes I+\mathscr{L} \\
& \mathscr{L}=\int_{0}^{\infty} \oplus \mathscr{L}(r) d r, \quad \mathscr{L}(r)=-\frac{1}{r^{2}} \frac{\partial^{2}}{\partial \phi^{2}}-\frac{1}{4 r^{2}} .
\end{aligned}
$$

The operator $\mathscr{L}(r)$ varies with the parameter $r \in \mathbb{R}_{+}$and acts in the space $\mathscr{F}_{r}=L^{2}[-\pi / 6, \pi / 6]$ on the domain

$$
\operatorname{Dom}(\mathscr{L}(r))=\mathscr{F}_{r} \cap\left\{f:-\left.\frac{1}{r} \frac{\partial f}{\partial \phi}\right|_{\phi= \pm \pi / 6}= \pm\left. g f\right|_{\phi= \pm \pi / 6}\right\} .
$$


This construction corresponds to the Hilbert bundle with the base space $\mathscr{M}=\mathbb{R}_{+}$ and Hilbert fibre $\mathscr{F}_{r}$. The frame Hamiltonian $\mathscr{L}(r)$ is self-adjoint on the domain $\operatorname{Dom}(\mathscr{L}(r))$ and thus its eigenfunctions $\varphi_{m}(\phi, r)$,

$$
\mathscr{L}(r) \varphi_{m}(\phi, r)=\lambda_{m}(r) \varphi_{m}(\phi, r),
$$

form the orthogonal basis (moving frame) in $\mathscr{F}_{r}$. This fact allows representation of the arbitrary function $v \in \mathfrak{H}$ in the form of triangle (adiabatic) expansion:

$$
v(r, \phi)=\sum_{m} \varphi_{m}(\phi, r) \chi_{m}(r)
$$

Hence (see Eq. (74)),

$$
u(r, \phi)=\frac{1}{\sqrt{r}} \sum_{m} \varphi_{m}(\phi, r) \chi_{m}(r)
$$

The spectral problem (77) for the instantaneous Hamiltonian $\mathscr{L}(r)$ can be exactly solved. The operator $\mathscr{L}(r)$ for every $r$ has the discrete spectrum only. The energy levels

$$
\lambda_{m}(r)=\frac{1}{r^{2}}\left(k_{m}^{2}(r)-\frac{1}{4}\right)
$$

vary with $r$ and are shown in Fig. 2.

The case $g>0$ corresponds to the delta-functional repulsion in the pair subsystem, and the case $g<0$, to the delta-functional attraction. The first situation is easier, because there are no two-body and three-body bound states. Here we consider the situation $g>0$ in detail and for $g<0$ we demonstrate the results only.

In the case $g>0$ we have two series of solutions of the spectral problem (77). One of them is

$$
\begin{aligned}
\varphi_{2 m}(\phi, r) & =w_{2 m}(r) \cos k_{2 m}(r) \phi, \quad m=1,2, \ldots \\
w_{2 m}(r) & =\left[\pi / 6\left(\sin \frac{\pi k_{2 m}(r)}{3}\right) / k_{2 m}(r)\right]^{-1 / 2}
\end{aligned}
$$

and $k_{2 m}(r)$ is the solution of the equation

$$
k_{2 m}(r)=r g \cot \frac{\pi k_{2 m}(r)}{6}
$$

Another series is

$$
\varphi_{2 m+1}(\phi, r)=w_{2 m+1}(r) \sin k_{2 m+1}(r) \phi, \quad m=1,2, \ldots
$$

where

$$
w_{2 m+1}(r)=\left[\pi / 6-\left(\sin \frac{\pi k_{2 m+1}(r)}{3}\right) / k_{2 m+1}(r)\right]^{-1 / 2},
$$




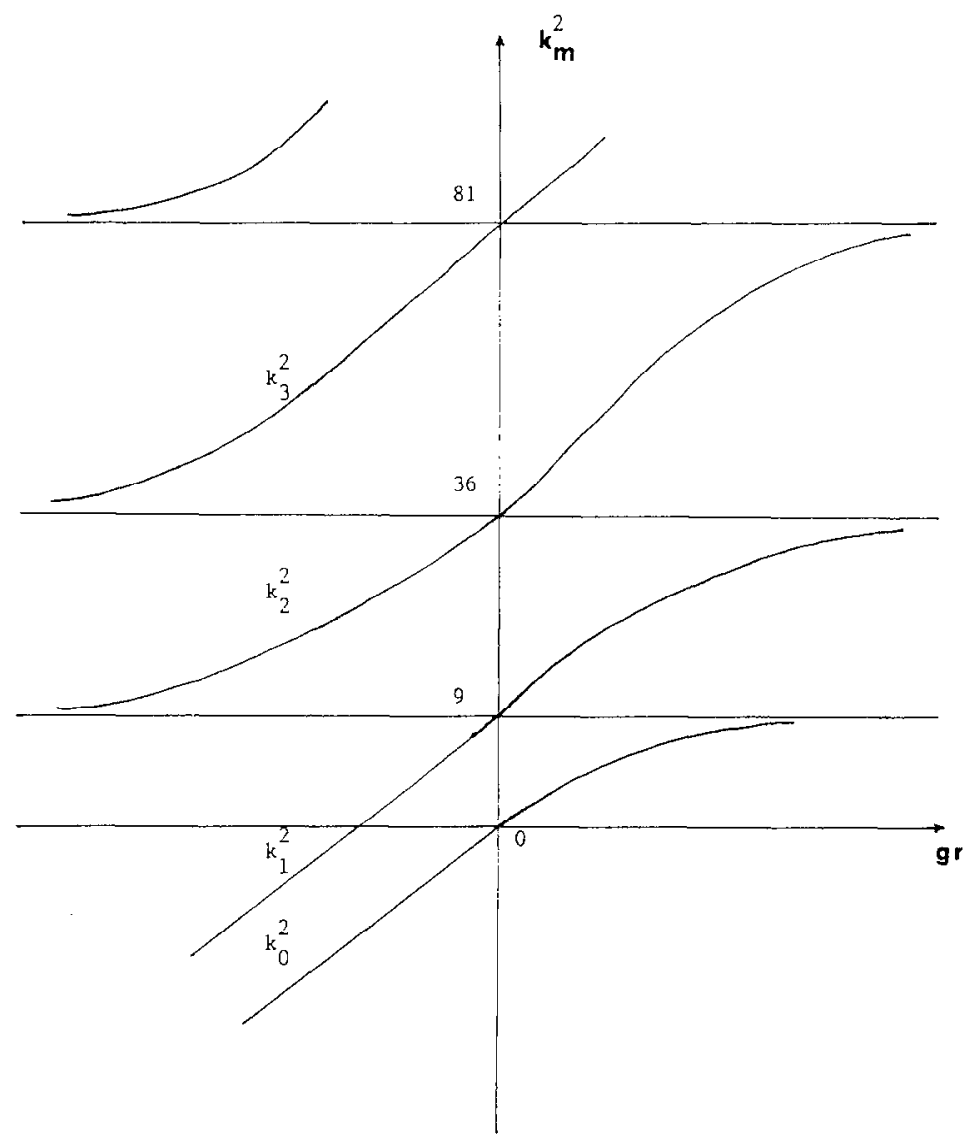

FIG. 2. Energy levels of the frame Hamiltonian $\mathscr{L}(x)$.

and $k_{2 m+1}(r)$ is the solution of the equation

$$
k_{2 m+1}(r)=-r g \tan \frac{\pi k_{2 m+1}(r)}{6} \text {. }
$$

\subsection{Effective Equation, Connection, and S-matrix}

According to the general scheme of the method (see Section 2), we substitute expansion (78) into Eq. (75) and consider the projection of the result on the eigenfunctions $\varphi_{m}(\phi, r)$ of the frame Hamiltonian $\mathscr{L}(r)$ in the space $\mathscr{F}_{r}=L^{2}(-\pi / 6, \pi / 6)$. The properties of completeness and orthogonality allow to obtain the result of the procedure mentioned above,

$$
\left[-\left(\frac{\partial}{\partial r} \otimes I+A\right)^{2}+A(r)-z\right] \chi=0
$$


wherc $A(r)=\operatorname{diag}\left\{\lambda_{m}(r)\right\}$ and $\chi$ is the element of the space

$$
\mathscr{H}=L^{2}\left(\mathbb{R}_{+}, l_{2}^{+}\right)
$$

The connection operator $A$ acts in the space $\mathscr{H}$ as the operator of multiplication by the operator-valued function $A(r)$,

$$
A=I_{r} \otimes A(r)
$$

where $I_{r}$ is the identical operator in $L^{2}\left(\mathbb{R}_{+}\right)$and the operator $A(r)$ acts in $l_{2}^{+}$are defined by their matrix elements:

$$
A_{n m}(r)=\int \frac{\partial \varphi_{m}}{\partial r} \overline{\varphi_{n}} d \phi
$$

The values $A_{n m}(r)$ can be exactly calculated, for example,

$$
\begin{aligned}
A_{2 n 2 m}(r)= & -w_{2 m} w_{2 n} \frac{\partial k_{2 m}}{\partial r}\left\{\frac { \pi } { 6 } \left[\frac{\cos \left(k_{2 n}-k_{2 m}\right)(\pi / 6)-1}{k_{2 n}-k_{2 m}}\right.\right. \\
& \left.-\frac{\cos \left(k_{2 n}+k_{2 m}\right)(\pi / 6)}{k_{2 n}+k_{2 m}}\right]+\frac{\sin \left(k_{2 n}+k_{2 m}\right)(\pi / 6)-\left(k_{2 n}+k_{2 m}\right)(\pi / 6)}{\left(k_{2 n}+k_{2 m}\right)^{2}} \\
& \left.-\frac{\sin \left(k_{2 n}-k_{2 m}\right)(\pi / 6)-\left(k_{2 n}-k_{2 m}\right)(\pi / 6)}{\left(k_{2 n}-k_{2 m}\right)^{2}}\right\}
\end{aligned}
$$

Let us notice, that analysis of Eqs. (80)-(86) allows us to obtain the following asymptotics of $A_{n m}(r)$ :

$$
A_{m n}(r) \cong \frac{\text { const }}{n m^{2}}, \quad m, n \rightarrow \infty .
$$

It means, that for every $r \in \mathbb{R}_{+}$, the operator $A(r)$ is a Hilbert-Schmidt operator in $l_{2}^{+}$.

Thus the triangle expansion (78) turns the spectral problem (75) in the space $\mathfrak{S}=L_{2}(\Phi)$ into the effective probleem $(84)$ in the space $\mathscr{H}=L_{2}\left(\mathbb{R}_{+}, l_{2}^{+}\right)$. Let us formulate the scattering problem for the effective operator

$$
\hat{H}=-\left(\frac{\partial}{\partial r} \otimes I+A\right)^{2}+\Lambda(r)
$$

acting in $\mathscr{H}$. In order to do that we consider the "asymptotical" effective operator

$$
\hat{H}_{0}=-\left(\frac{\partial}{\partial r} \otimes I+I_{r} \otimes A_{\infty}\right)^{2}+I_{r} \otimes A_{\infty},
$$

where $A_{\infty}, A_{\infty}$ are limits of $A(r), A(r)$ at $r \rightarrow \infty$ for every component: $\left(A_{\infty}\right)_{m n}=$ 
$\lim _{r \rightarrow \infty} A_{m n},\left(A_{\infty}\right)_{m n}=\lim _{r \rightarrow \infty} A_{m n}$. The analysis of Eqs. (80) (86) gives the following asymptotic behaviour of the elements $A_{m n}(r), A_{m n}(r)=\delta_{m n} \lambda_{m}(r)$ :

$$
\begin{gathered}
A_{m n}(r) \underset{r \rightarrow \infty}{\cong} \frac{c_{m n}}{g} \frac{1}{r^{2}}+O\left(r^{-3}\right), \\
\lambda_{m}(r) \underset{r \rightarrow \infty}{\cong} \frac{A_{m}}{r^{2}}+O\left(r^{-3}\right) .
\end{gathered}
$$

Thus the limits $A_{\infty}$ and $A_{\infty}$ do exist and are equal to zero and the "asymptotical" effective Hamiltonian looks like

$$
\hat{H}_{0}=-\frac{\partial^{2}}{\partial r^{2}} \otimes I
$$

We consider the Hamiltonian $\hat{H}_{0}$ on the domain $\operatorname{Dom}\left(\hat{H}_{0}\right)=\mathscr{H} \cap\left\{\chi:\left.\chi\right|_{r=0}=0\right\}$ as the nonperturbed operator. The eigenfunctions $\chi^{a s}$ are

$$
x^{a s}(r)=\left(e^{-i \sqrt{z} r}-e^{i \sqrt{z} r}\right) J,
$$

where $J$ is the arbitrary vector $J \in l_{2}^{+}$. Solution of the effective spectral problem (84) for the perturbed Hamiltonian $\hat{H}$ has the asymptotics:

$$
\chi(r)=e^{-i \sqrt{z} r} J_{\text {inc }} \quad e^{i \sqrt{z} r} J_{\text {out }} .
$$

We define the effective $S$-matrix for the pair of the operators $\hat{H}_{0}, \hat{H}$ as

$$
J_{\text {out }}=S^{\text {eff }}(z) J_{\text {inc }}
$$

for arbitrary $J_{\text {inc }} \in l_{2}^{+}$. The effective scattering matrix defincd by Eq. (88) is the operator in the space $l_{2}^{+}$:

$$
S^{\mathrm{eff}}(z)=\left\{S_{m n}^{\mathrm{eff}}(z)\right\}_{m, n=0}^{\infty} .
$$

Now we obtain the relations between $S^{\text {eff }}(z)$ and the global $S$-matrix of the original scattering problem in the sector $\Phi$. In the case $g>0$ (repulsion) the global $S$-matrix has the only non-trivial matrix elements $S_{33}$, corresponding to the process $3 \rightarrow 3$. It can be defined as the operator which turns the amplitude at the incoming spherical wave in $\mathbb{R}^{2}$ into that of the outgoing spherical wave. Namely, the solution $u(r, \phi)$ of the spectral problem $(76)$ has the asymptotics

$$
u(r, \phi) \underset{r \rightarrow \infty}{\cong} \frac{e^{-i \sqrt{z} r}}{\sqrt{r}} \mathfrak{J}^{\text {inc }}(\phi)+\frac{e^{i \sqrt{z} r}}{\sqrt{r}} \mathfrak{J}^{\text {out }}(-\phi) .
$$

It is more convenient to use $\mathfrak{J}^{\text {out }}(-\phi)$ in Eq. (89) in order not to consider the inversion operator [28]. We refer to the operator

$$
S_{33}: \mathfrak{J}^{\text {inc }}(\phi) \rightarrow \mathfrak{J}^{\text {out }}(\phi)
$$


with the kernel $\hat{\mathscr{S}}_{33}\left(\phi, \phi^{\prime}, z\right)$ as the scattering matrix for the $3 \rightarrow 3$ scattering in the sector $\Phi$. Using the triangle representation (79), asymptotics (87), and definition (88), one can obtain the asympotical representation for the function $u(r, \phi)$,

$$
u(r, \phi) \underset{r \rightarrow \infty}{\cong} \frac{e^{-i \sqrt{z} r}}{\sqrt{r}} \sum_{m} J_{m} \varphi_{m}^{\infty}(\phi)-\frac{e^{i \sqrt{z} r}}{\sqrt{r}} \sum_{m} \varphi_{m}^{\infty}(\phi) \sum_{n} S_{m n}^{\mathrm{eff}} J_{n},
$$

where $J_{m}$ are the components of the vector $J_{\text {inc }}$ and $\varphi_{m}^{\infty}(\phi)$ are the following limits:

$$
\varphi_{m}^{\infty}(\phi)=\lim _{r \rightarrow \infty} \varphi_{m}(r, \phi) \text {. }
$$

One can see that

$$
\begin{aligned}
\varphi_{2 m}^{\infty}(\phi) & =\sqrt{6 / \pi} \cos [3(2 m+1) \phi], \\
\varphi_{2 m+1}^{\infty}(\phi) & =\sqrt{6 / \pi} \sin [6(m+1) \phi] .
\end{aligned}
$$

Comparing the asymptotics and using the orthogonality property, we have the relations,

$$
\begin{aligned}
-\hat{\mathscr{S}}_{33}\left(\phi, \phi^{\prime}, z\right) & =\sum_{m} \sum_{n} S_{m n}^{\mathrm{eff}}(z) \varphi_{m}^{\infty}(-\phi) \overline{\varphi_{n}^{\infty}\left(\phi^{\prime}\right)}, \\
-S_{m n}^{\mathrm{eff}}(z) & =\int d \phi \int d \phi^{\prime} \hat{\mathscr{Y}}_{33}\left(-\phi, \phi^{\prime}, z\right) \overline{\varphi_{m}^{\infty}(\phi)} \varphi_{n}^{\infty}\left(\phi^{\prime}\right) .
\end{aligned}
$$

Relations (90) and (91) are of general character and realize the relations between the operators $\tilde{H}$ and $\hat{H}$ in terms of scattering matrices. Relations (90), (91) connect the total and effective $S$-matrices.

However, the model under discussion allows us to obtain more information about these relations and gives an example of an exactly calculated $S$-matrix for the matrix Schroedinger operator $\hat{H}$ in the space $\mathscr{H}=L^{2}\left(-\pi / 6, \pi / 6, l_{2}^{1}\right)$. Investigation of the three-body scattering problem in one dimension by the Sommerfeld integral method [22-26] gives an opportunity to obtain the exact expression for the kernel $\mathscr{F}_{33}\left(\phi, \phi^{\prime}, z\right)$,

$$
\hat{\mathscr{S}}_{33}\left(\phi, \phi^{\prime}, z\right)=B(\pi / 3-\phi, z) B(\phi, z) B(\pi / 3+\phi, z) \delta\left(\phi-\phi^{\prime}\right),
$$

where

$$
B(\alpha, z)=\frac{i \sqrt{z} \cos \alpha+g}{i \sqrt{z} \cos \alpha-g} .
$$

Hence, using Eq. (91), we have

$$
S_{m n}^{\mathrm{eff}}(z)=\int B(\pi / 3-\phi) B(\phi) B(\pi / 3+\phi) \varphi_{m}^{\infty}(\phi) \varphi_{n}^{\infty}(-\phi) d \phi .
$$


Let us point out some facts which are important from our point of view.

(1) The effective equation (84) is a matrix ordinary differential equation. The methods of solution of the inverse problem for such equations are well known [27]. Using these methods one can try to reconstruct the effective potential, determined by the matrix-valued potentials $A(r)$ and $V(r)$ in terms of $S^{\text {eff }}(z)$ [12]. As the result one will obtain the relations which associate $S^{\text {eit }}(z)$ and, consequently $S_{33}(z)$ (see Eq. (91)) with the connection $A$ which plays the role of the gauge field in the effective equation (84).

(2) The model becomes more extensive in the case $g<0$ (attraction), where there exist 2-body and 3-body bound states. Thus the global $S$-matrix contains the matrix elements corresponding to $2 \rightarrow 2$ and $3 \rightarrow 3$ processes. However, it can be shown, that in the case of equal particle masses there are no $2 \rightarrow 3$ and $3 \rightarrow 2$ processes in the system. This fact is not very surprising and has the classical analogy: three elastic balls on the line. The "bound state" of two balls touching each other after being struck by the third ball turns into the "bound state" of the second and third balls with the first one moving freely. The diffraction on the system of three screens located symmetrically (which is absolutely analogous to the system of three one-dimensional particles) gives another example of the same phenomenon. Let us notice, that processes $2 \rightarrow 3$ and $3 \rightarrow 2$ for three-point one-dimensional particles of equal masses can be included in consideration, if one considers any kind of complicated internal structure of the particles [26]. In our model if $g<0$, we have only the $S_{22}$ matrix element of the scattering matrix besides $S_{33}$. If one denotes as $f_{\text {inc }}^{ \pm}$ the amplitudes of incoming plane waves propagating along the screens $\phi= \pm \pi / 6$ (see Fig. 1) and as $f_{\text {out }}^{ \pm}$the amplitudes of outgoing ones, and the $S_{22}$ element of the scattering matrix is defined

$$
\left(\begin{array}{l}
f_{\text {out }}^{+} \\
f_{\text {out }}^{-}
\end{array}\right)=S_{22}\left(\begin{array}{l}
f_{\text {inc }}^{+} \\
f_{\text {inc }}^{-}
\end{array}\right)
$$

we can ensure that $S_{22}$ has the antidiagonal form

$$
S_{22}(z)=\left(\begin{array}{cc}
0 & T(z) \\
T(z) & 0
\end{array}\right)
$$

This representation means that we have no reflection of the two-body plane waves in our model. The transition coefficient $T(z)$ can be calculated as

$$
T(z)=B(\pi / 3-\tau(z), z) B(\tau(z), z),
$$

where $\tau(z)$ satisfies the equation

$$
i \sqrt{z} \cos (\pi / 3+\tau(z))=g
$$

and $B(\alpha, z)$ is defined by Eq. (92). Let us notice, that $T(z)$ has the only singularity 
at $z=-g^{2}$ which corresponds to the energy of the three-body bound state whose eigenfunction is

$$
u_{0}(\phi, r)=\exp (2 g r \cos \phi) .
$$

On the other hand, the existence of bound states in the triangle representation approach means the existence of negative energy levels of the frame Hamiltonian $\mathscr{L}(r)$ (see Fig. 2). The corresponding eigenfunctions $\varphi_{0}^{\infty}(\phi)$ and $\varphi_{1}^{\infty}(\phi)$ generate the matrix elements of the effective $S$-matrix $S^{\text {eff }}(z)$, associated with $S_{22}$ in the representation, analogous to (90) and (91).

(3) A more comprehensive exactly solvable model with non-trivial connections of geometrical ingredients of the Hilbert bundle and spectral characteristics of the Hamiltonian can be obtained by consideration of the three one-dimensional particles with an internal structure [26]. Of main interest in this situation is the interaction of spectra of internal (simulating the internal structure) and external (three-body) Hamiltonians displayed in the corresponding geometrical objects. This problem is still under consideration.

\section{Wave Propagation by a Curved Quantum Waveguide}

\subsection{Curvilinear Coordinates and the Triangle Representation}

In this section we represent one more example of the application of the triangle expansion method to a quantum mechanical problem. We study the system whose dynamics is determined by Schroedinger equation in curved planar strip $\Omega$ of a fixed width $d$ with some self-adjoint boundary conditions varying along the strip. It can be serve as a model for clcctrons in the film or in a curved quantum wire [29]. We call such a system a curved quantum waveguide [31-35]. We denote as $\partial \Omega_{+}=$

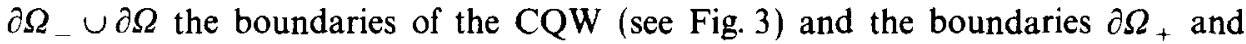
$\partial \Omega_{-}$are assumed to be identical, i.e., the boundary conditions on $\partial \Omega_{+}$and $\partial \Omega_{-}$ coincide. Thus we have the following boundary problem in configuration space $\Omega$ :

$$
\begin{aligned}
-\Delta \Psi & =z \Psi \\
\left.\partial_{n} \Psi\right|_{\partial \Omega_{ \pm}} & =\left.\gamma(s) \Psi\right|_{\partial \Omega_{ \pm}}, \quad \gamma(s) \in \mathbb{R} .
\end{aligned}
$$

Here $\partial_{n}$ stands for the internal normal derivative (see Fig. 3 ).

Let us introduce the local coordinates $(v, s)$, associated with the strip $\Omega$. The transformation into the local coordinates $(v, s)$ determines the metric in $\mathbb{R}^{2}$ :

$$
d^{2} x+d^{2} y=g_{s s} d s^{2}+g_{v v} d v^{2},
$$

where $(x, y)$ are Cartesian coordinates and the components of the metric tensor are

$$
\begin{aligned}
& g_{s s} \equiv g=(1+v C(s))^{2}, \\
& g_{v v}=1 .
\end{aligned}
$$




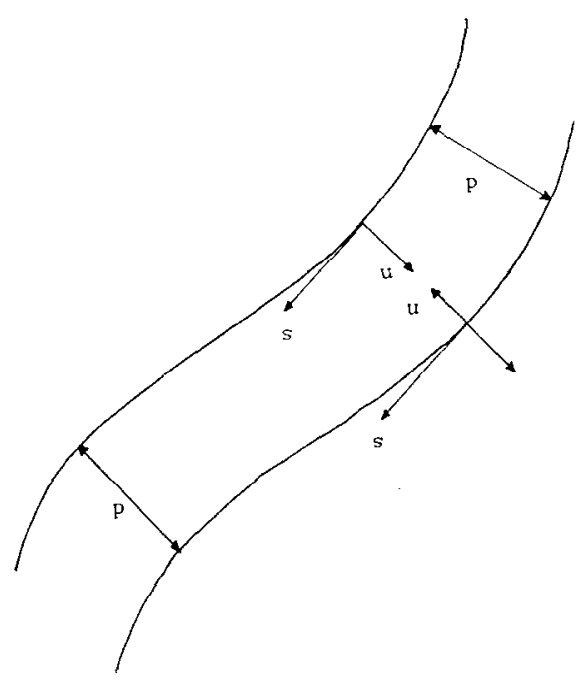

FIG. 3. The waveguide $\Omega$.

Here $C(s)$ is the curvature of the curves $\partial \Omega_{ \pm}$. The Jacobian of the transformation into the local coordinates $(v, s)$ looks like

$$
\frac{D(x, y)}{D(v, s)}=1+v C(s)=g^{1 / 2}
$$

The change of variables $(x, y) \mapsto(v, s)$ is regular in every point inside $\Omega$ if $d<|C(s)|^{-1}, \forall s \in \mathbb{R}$. The Laplace operator $\Delta=\partial^{2} / \partial x^{2}+\partial^{2} / \partial y^{2}$ in the coordinates $(v, s)$ has the form

$$
\begin{aligned}
\Delta & =g^{-1 / 2} \frac{\partial}{\partial s} g^{-1 / 2} \frac{\partial}{\partial s}+g^{-1 / 2} \frac{\partial}{\partial v} g^{1 / 2} \frac{\partial}{\partial v} \\
& =(1+v C(s))^{-1}\left\{\frac{\partial}{\partial v}(1+v C(s)) \frac{\partial}{\partial v}+\frac{\partial}{\partial s}(1+v C(s))^{-1} \frac{\partial}{\partial s}\right\}
\end{aligned}
$$

Henceforth we suppose the curvature $C(s)$ to be small. Namely, the conditions

$$
d k \ll 1 \ll k / C, \quad C=\max _{s}|C(s)|,
$$

are assumed, where $k$ is the wave number. Under these conditions we can approximate the Laplace operator $\Delta$ by the operator

$$
\Delta_{\varepsilon}=\partial^{2} / \partial s^{2}+C(s) \partial / \partial v+\partial^{2} / \partial v^{2}
$$


This approximation is quite reasonable in this region of energies, $z=k^{2}$. Now we consider the approximate boundary problem

$$
\begin{aligned}
-\Delta_{\varepsilon} \Psi & =z \Psi \\
\left.\partial_{\nu} \Psi\right|_{v=0} & =\left.\gamma(s) \Psi\right|_{\nu=0} \\
\left.\partial_{v} \Psi\right|_{\nu=d} & =-\left.\gamma(s) \Psi\right|_{\nu=d}
\end{aligned}
$$

for the operator $-\Delta_{\varepsilon}$ in the Hilbert space

$$
\mathfrak{H}=L^{2}\left([0, d] \times \mathbb{R}_{5}, \rho_{s}^{2}(v) d v d s\right),
$$

where $\rho_{s}(v)=\exp \left\{\frac{1}{2} C(s) v\right\}, \partial_{v} \equiv \partial / \partial v$.

The self-adjoint operator $-\Delta_{\varepsilon}$ can be represented in the form

$$
-\Delta_{\varepsilon}=-\partial^{2} / \partial s^{2}+\int_{-\infty}^{\infty} \oplus \mathscr{L}_{\varepsilon}(s) d s,
$$

where the frame operator is

$$
\mathscr{L}_{\varepsilon}(s)=-\partial^{2} / \partial v^{2}-C(s) \partial / \partial v .
$$

The eigenvalue problem (96) generates the Hilbert bundle over the one-dimensional base space $\mathbb{R}_{s}$ with Hilbert fibres $\mathscr{F}_{s}=L^{2}\left([0, d], \rho_{s}^{2}(v) d v\right)$. The following lemma is valid.

LEMMA 9. The operator $\mathscr{L}_{\varepsilon}(s)$ is self-adjoint on the domain

$$
\begin{aligned}
\mathscr{D}\left(\mathscr{L}_{\varepsilon}(s)\right)= & W_{2, \rho_{s}}^{2}(0, d) \cap\left\{\psi(v):\left.\partial_{v} \psi\right|_{v=0}=\gamma(s) \psi(0),\right. \\
& \left.\left.\partial_{v} \psi\right|_{\nu=d}=-\gamma(s) \psi(d)\right\} \\
& \text { in the Hilbert space } L_{\rho_{s}}^{2}\left(\mathbb{R}_{v}\right)
\end{aligned}
$$

with the inner product

$$
\left\langle u, v 》=\int_{0}^{d} u(v) \overline{v(v)} \rho_{s}^{2}(v) d v .\right.
$$

This lemma allows application of the general scheme of the triangle expansion method described in Section 2 to CQW. Namely, one can represent the solution of the eigenvalue problem (96) in the form

$$
\psi=\sum_{m} \chi_{m}(s) \varphi_{m}(v, s)
$$

where $\varphi_{m}(v, s)$ are the eigenfunctions of the frame Hamiltonian $\mathscr{L}_{\varepsilon}(s)$. The effective equations (12) read here as

$$
\left[-\left(\partial_{s}+A(s)\right)^{2}+A(s)-z\right] \chi(s)=0,
$$


where $A_{m n}(s)=\left\langle\partial_{s} \varphi_{n}, \varphi_{m}\right\rangle, \Lambda(s)=\operatorname{diag}\left\{\lambda_{m}(s)\right\}$, and $\lambda_{m}(s)$ are the eigenvalues of the frame operator $\mathscr{L}_{\varepsilon}(s)$.

\subsection{The Frame Problem for $C Q W$}

The eigenvalue problem

$$
\mathscr{L}_{\varepsilon}(s) \varphi_{m}(v, s)=\lambda_{m}(s) \varphi_{m}(v, s)
$$

for the frame operator $\mathscr{L}_{\varepsilon}(s)$ by the following change of variables $\psi(v, s) \equiv$ $\rho_{s}(v) \varphi(v, s)$ becomes

$$
\begin{aligned}
-\partial_{v}^{2} \psi_{m}(v, s) & =k_{m}^{2}(s) \psi_{m}(v, s) \\
\left.\partial_{v} \psi_{m}\right|_{v=0} & =\gamma(s) \psi_{m}(0, s) \\
\left.\partial_{v} \psi_{m}\right|_{v=d} & =-\gamma(s) \psi_{m}(d, s),
\end{aligned}
$$

where

$$
k_{m}^{2}(s)=\lambda_{m}(s)-\frac{1}{4} C^{2}(s)
$$

This change of variables transforms the metric of the space,

$$
L_{\rho_{s}}^{2}\left(\mathbb{R}_{v}\right) \rightarrow L^{2}\left(\mathbb{R}_{v}\right)
$$

and its eigenvalues are given by the roots of the equation

$$
k^{2}(s)+\frac{1}{4} C^{2}(s)=2 k(s) \gamma(s) \cot (k(s) d)+\gamma^{2}(s)
$$

Their behaviour is very similar to that of the levels in Fig. 2 and there is no level crossing. The corresponding eigenfunctions have the form

$$
\begin{aligned}
\psi_{m}(v, s)=[ & \left.d\left(\gamma^{2}(s)+k_{m}^{2}(s)\right) / 2\right]^{-1 / 2}\left\{\gamma(s) \sin \left(k_{m}^{2}(s) v\right)\right. \\
& \left.+k_{m}(s) \cos \left(k_{m}^{2}(s) v\right)\right\}, \quad m=0,1,2, \ldots
\end{aligned}
$$

One can see, that if $\gamma(s) \in C^{2}(\mathbb{R})$, then the eigenfunctions $\varphi_{m}(v, s)$ have two derivatives over the parameter $s$ and thus the formal scheme of the triangle expansions method is justified.

The straightforward calculation of the matrix elements of the connection operator $A$ gives

$$
\begin{aligned}
A_{n m}(s) \equiv & \left\langle\partial_{s} \varphi_{m}, \varphi_{n}\right\rangle \\
= & \frac{1}{2} N_{m} N_{n}\left(\frac{\sin \left(d\left(k_{m}-k_{n}\right)\right)}{k_{m}-k_{n}}\left[\gamma\left(\partial_{s} \gamma-k_{m} \partial_{s} k_{m}\right)+\partial_{s} k_{m} k_{n}(\gamma+1)+\gamma \partial_{s} C(s)\right]\right. \\
& +\frac{\sin \left(d\left(k_{m}+k_{n}\right)\right)}{k_{m}+k_{n}}\left[\gamma\left(\partial_{s} \gamma-k_{m} \partial_{s} k_{m}\right)\right. \\
& \left.+\partial_{s} k_{m} k_{n}(\gamma+1)-\gamma \partial_{s} C(s)\right]
\end{aligned}
$$




$$
\begin{aligned}
& +\frac{\cos \left(d\left(k_{m}-k_{n}\right)\right)-1}{k_{m}-k_{n}}\left[k_{n}\left(\partial_{s} \gamma-k_{m} \partial_{s} k_{m}\right)-\partial_{s} k_{m} \gamma(\gamma+1)\right] \\
& +\frac{\cos \left(d\left(k_{m}+k_{n}\right)\right)-1}{k_{m}+k_{n}}\left[-k_{n}\left(\partial_{s} \gamma-k_{m} \partial_{s} k_{m}\right)-\partial_{s} k_{m} \gamma(\gamma+1)\right] \\
& \left.-\left(\gamma^{2}-k_{m} k_{n}\right) \partial_{s} C(s)\left[\frac{\cos \left(d\left(k_{m}-k_{n}\right)\right)-1}{\left(k_{m}-k_{n}\right)^{2}}-\frac{\cos \left(d\left(k_{m}+k_{n}\right)\right)-1}{\left(k_{m}+k_{n}\right)^{2}}\right]\right),
\end{aligned}
$$

where

$$
N_{m}(s)=\left[d\left(\gamma^{2}(s)+k_{m}^{2}(s)\right) / 2\right]^{-1 / 2}
$$

An analysis similar to that of Section 4 gives

$$
A_{n m}(s)=\frac{\text { const }}{m n}(1+o(1)) \quad \text { at } m, n \rightarrow \infty
$$

Hence $A(s)$ is Hilbert-Schmidt operator in $l_{2}^{+}$at every $s$.

\subsection{The Inverse Problem: A Scheme of Solution}

Now we are going to use a step-by-step procedure based on the separation of the frame problem (100) from the total original problem (96) and the corresponding reduction to the the effective problem (99), for the reconstruction of the functions $\gamma(s), C(s)$ in terms of scattering data. This procedure allows us to understand the role of the effective gauge field $A$ as the coupling operator between scattering data of the effective problem and the frame problem.

In order to define the scattering data for the effective problem (75) let us suppose the following asymptotical behaviour of the curve $\partial \Omega_{ \pm}$:

$$
\begin{array}{lll}
C(s) \rightarrow 0 & \text { when } & s \rightarrow \pm \infty, \\
\gamma(s) \rightarrow \gamma_{\infty}=\mathrm{const} & \text { when } & s \rightarrow \pm \infty .
\end{array}
$$

The effective equation (99) can be rewritten using Lemma 1 in the form

$$
\left[-d^{2} / d s^{2}+A(s)-\left(A^{2}(s)+d A(s) / d s\right)-2 A(s) d / d s\right] \chi=z \chi
$$

Let us denote $\hat{\chi}(s)=\left(\chi^{(1)}, \chi^{(2)}\right)$, where $\chi^{(1)}=d x / d s, \chi^{(2)}=\chi(s)$ and rewrite Eq. (107) in the form

$$
d / d s \hat{\chi}+\mathscr{W}(s, z) \hat{\chi}=0
$$

where

$$
\mathscr{W}(s, z)=\left(\begin{array}{cc}
2 A & A^{2}+d A / d s+(z-A) \\
-I & 0
\end{array}\right)
$$


Let us consider the problem with the "asymptotical" matrix potential $\mathscr{W}_{\infty}(z)=$ $\lim _{s \rightarrow \pm \infty} \mathscr{W}(s, z)$ as the non-perturbed one:

$$
d / d s \hat{\chi}+\mathscr{W}_{\infty}(z) \hat{\chi}=0 .
$$

The diagonal operator $\Lambda(s)$ has the matrix elements $\lambda_{m}(s)$, given by Eq. (102).

Due to the assumption (105), the "asymptotical" matrix potential $\mathscr{W}_{\infty}(z)$ has the form

$$
\mathscr{W}_{\infty}(z)=\left(\begin{array}{cc}
0 & z-\Lambda_{\infty} \\
-I & 0
\end{array}\right) .
$$

Here, due to the assumption (100),

$$
\Lambda_{\infty}=\left\{\lambda_{m}^{\infty}\right\}, \quad \lambda_{m}^{\infty}=\lim _{s \rightarrow \infty} k_{m}^{2}(s) .
$$

Let us denote as $\hat{\chi}_{J}^{ \pm}(s, z)$ the solutions of the Eq. (84), determined by the asymptotics

$$
\hat{\chi}_{J}^{ \pm}(s, z) \rightarrow \hat{\chi}_{J}^{\text {as }}(s, z) \quad \text { when } \quad s \rightarrow \pm \infty,
$$

where $\hat{\chi}^{\text {as }}(s, z)$ are the following solutions of the non-perturbed equation (110):

$$
\hat{\chi}_{J}^{\text {as }}(s, z)=\left(\begin{array}{l}
i \mathscr{P}(E) \exp (i s \mathscr{P}(z)) \circ J \\
\exp (i s \mathscr{P}(z)) \circ J
\end{array}\right) .
$$

In Eq. (113),

$$
\mathscr{P}(z)=\left(z I-A_{\infty}\right)^{1 / 2}
$$

and $J \in l_{2}^{+}$. One can check that $\hat{\chi}^{\text {as }}(s, z)$ is the solution of the non-perturbed equation (110). We define the scattering matrix $S^{\text {eff }}(z)$ of the effective problem (99) (i.e., of Eq. (108)) as follows:

$$
\hat{\chi}_{J}^{+}(0, z)=S^{\mathrm{eff}}(z) \hat{\chi}_{J}^{-}(0, z) .
$$

Equation (108) is an ordinary linear differential equation with the matrix potential $\mathscr{W}(s, z)$. For such equations the inverse problem can be solved [27] and thus

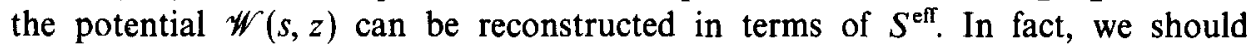
consider the finite-dimensional block of $S^{\text {eff }}$ only, because the contribution to $S^{\text {eff }}$ is given only by propagating normal modes, corresponding to $\varphi_{m}$ with $m<m_{0}$. The value $m_{0}$ is determined by the width $d$ of the CQW and its curvature $C(s)$.

According to Eq. (109), $\mathscr{W}(s, z)$ has the structure

$$
\mathscr{W}(s, z)=\left(\begin{array}{cc}
\mathscr{W}^{11}(s, z) & \mathscr{W}^{12}(s, z) \\
-I & 0
\end{array}\right)
$$


where $\mathscr{W}^{i j}(s, z)$ are infinite-dimensional operators. Equations (109) and (115) give

Hence

$$
\mathscr{W}^{11}=2 A, \quad \mathscr{W}^{12}=A^{2}+d A / d s+(z-\Lambda) .
$$

$$
\Lambda(s)=\left[\frac{1}{4}\left(\mathscr{W}^{11}(s, z)\right)^{2}+\frac{1}{2} \partial \mathscr{W}^{11}(s, z) / \partial s-\mathscr{W}^{12}(s, z)\right]+z, \quad z \in \mathbb{R} .
$$

The frame Hamiltonian $\mathscr{L}_{\varepsilon}(s)$ has no continuous spectrum, and we refer to the set $\left\{S^{\text {eff }}, N_{m}\right\}$, where $N_{m}$ is the normalizing coefficient of the eigenfunction $\varphi_{m}$ (see Eq. (100)) for arbitrary fixed $m$, as the scattering data. We formulate the inverse problem as the problem of reconstructing $C(s)$ and $\gamma(s)$ in terms of scattering data.

The scheme of the inverse problem solution is the following:

(1) Using Eq. (117), we express $\lambda_{m}(s)$ in terms of $\mathscr{W}(s, z)$, i.e., in terms of the effective scattering matrix $S^{\text {eff }}(z)$ (the solution of the inverse problem for the effective Eq. (110) is not discussed here; see, e.g., [27]).

(2) Using Eqs. (102), (104), and (107) one obtains

$$
\begin{aligned}
\gamma^{2}(s)-\frac{1}{4} C^{2}(s)= & \frac{2}{d N_{m}^{2}(s)}-\left[\frac{1}{4}\left(\mathscr{W}^{11}(s, z)\right)_{m m}^{2}\right. \\
& \left.+\frac{1}{2} \partial \mathscr{W}_{m m}^{11}(s, z) / \partial s-\mathscr{W}_{m m}^{12}(s, z)\right]-z
\end{aligned}
$$

In order to reconstruct two unknown functions $C(s)$ and $\gamma(s)$, one needs two independent equations. One of them is given by the expression (118). In order to obtain the second equation, one should solve Eq. (103) and express the function $C(s)$ in terms of $k_{m}^{2}(s)$ and $\gamma(s)$. We denote such a solution of Eq. (103) as

$$
C(s) \equiv T_{m}\left(k_{m}^{2}, \gamma, s\right)=T_{m}\left(\frac{2}{d N_{m}^{2}(s)}-\gamma^{2}(s), \gamma(s), s\right) .
$$

The system of Eq. (118) and (119) allows us to find two functions $C(s)$ and $\gamma(s)$.

\section{ACKNOWLEDGMENTS}

Particular thanks are due to Professor L. D. Faddeev for encouragement and helpful suggestions throughout the course of this work. One of us (YuAK) is indebted to Professor C. Ciofi degli Atti, Istituto Nazionale di Fisica Nucleare, for warm hospitality. We are also grateful to Professor B. S. Pavlov for many fruitful discussions.

\section{REFERENCES}

1. K. A. Makarov, Yu. A. Kuperin, B. S. Pavlov, V. M. Dubovik, B. L. Markovski, and S. I. VINITSKy, "A Local Adiabatic Representation in the Few-Body Quantum Scattering Problem," Preprint FUB-HEP/87-11, West Berlin, 1987.

2. B. ZygelmanN, Phys. Lett. A 125 (1987), 476. 
3. V. M. Dubovik, B. L. Markovski, And S. I. Vinitsky, "Multistep Adiabatic Representation," Preprint JINR E4-87-743, 1987.

4. J. M. Combes, P. Duclos, And R. Seiler, Commun. Math. Phys. 92 (1983), 229.

5. M. V. Berre, Proc. Roy. Soc. London 392 (1982), 45.

6. F. WilczeK AND A. ZeE, Phys. Rev. Lett. 52 (1984), 2111.

7. A. Niem and G. Semenoff, Phys. Rev. Lett. 55 (1985), 927.

8. E. Kiritsis, Commun. Math. Phys. 111 (1987), 417.

9. T. IWA1, J. Math. Phys. 28 (1987), 964.

10. V. M. Dubovik, Yu. A. Kuperin, B. L. Markovski, Yu. B. Melnikov, and S. I. Vinitsky, "Five-Body Adiabatic Treatment of $d+t \rightarrow n+\alpha$ Reaction," Preprint JINR P4-88-62, Dubna, 1988. [Russian]

11. YU. A. Kuperin, YU. B. Melnikov, and S. I. Yakovlev, Dimensional reduction of the few-body problem and moving frames asymptotics, in "Proceedings, International Few-Body Workshop, Microscopic Methods in the Theory of Few-Body Systems, Kalinin, Augsut 1988," Vol. 1, pp. 164-170.

12. Yu. A. Kuperin, Yu. B. Melnikov, and B. S. Pavlov, Quantum scattering problem in triangle representation and induced gauge fields, in "Proceedings, Internat. Workshop on Schroedinger Operators: Standard and Non-Standard, Dubna, September 6-10, 1988," World Scientific. Singapore, pp. 295-319.

13. A. Salam and S. StrathdeE, Ann. Phys. (N.Y.) 141 (1982), 1711.

14. J. Scherk and J. H. Schwarz, Phys. Lett. B 57 (1975), 463.

15. H. Feshbach, Ann. Phys. (N.Y.) 5 (1958), 357; 19 (1962), 287.

16. N. Steenrod, "The Topology of Fibre Bundles," Princeton Univ. Press, Princeton, NJ, 1951.

17. B. Kostant, "Lectures Notes in Mathematics," (T. Taam, Ed.), Vol. 170, p. 87, Springer-Verlag, New York/Berlin, 1970.

18. R. D. Walls, "Differential Analysis on Complex Manifolds," Prentice-Hall, Englewood Cliffs, NJ, 1973.

19. V. P. MASLov, "Asymptotical Methods in Perturbation Theory," Nauka, Moscow, 1988. [Russian]

20. J. E. AVRon, A. RaVksh, AND B. Zur, Phys. Rev. Lett. 58 (1987), 2110.

21. S. P. MerkuRiev and L. D. Faddeev, "Quantum Scattering Theory for Few-Body Systems," Nauka, Moscow, 1985. [Russian]

22. J. B. MCGuire, J. Math. Phys. 5 (1964), 622.

23. J. B. McGuire and C. A. Hurst, J. Math. Phys. 13 (1972), 1595.

24. V. S. Buslaev, S. P. Merkuriev, and S. P. Salikov, in "Problems of Mathematical Physics," Vol. 9, Leningrad Univ. Press, Leningrad, 1979. [Russian]

25. J. B. McGuire and C. A. Hurst, J. Math. Phys. 29 (1987), 155.

26. P. B. Kurasov, Three One-Dimensional Bosons with an Internal Structure," in "Proceedings, Internat. Workshop on Schroedinger Operators, Standard and Non-Standard, Dubna, September 6-10, 1988," World Scientific, Singapore, pp. 167-188.

27. P. S. Sabatier and K. Chadan, "Inverse Problems in Quantum Scattering Theory," SpringerVerlag, Berlin/Heidelberg/New York, 1977.

28. R. T. Pack and G. A. Parker, J. Chem. Phys. 87, No. 7 (1987), 3888.

29. P. Exner And P. Seba, "Bound States in Curved Quantum Waveguides," Preprint BiBoS 298/87, Bielefeld, 1987.

30. W. B. Gibson, S. Y. Larsen, and P. Papiel, Phys. Rev. 35 (1987), 4919.

31. B. Z. Kantsenelenbaum, "Theory of Non-Regular Waveguides with Weakly Varying Parameters," Nauka, Moscow, 1961. [Russian]

32. L. Levin, "Theory of Waveguides," Nauka, Moscow, 1981. [Russian]

33. B. M. Mashkovtsev, K. N. Tsibizov, aND B. F. Emelin, "Theory of Waveguides," Nauka, Moscow/Leningrad, 1966. [Russian]

34. A. G. Sveshnikov, Soviet J. Comput. Math. Math. Phys. 3, No. 1 (1963), 170; No. 2 (1963), 314.

35. M. V. FedoriuK, Soviet J. Comput. Math. Math. Phys. 13, No. 1 (1973), 127.

36. S. P. Merkuriev, Yad. Fiz. 19 (1974), 447. 